\title{
On the Behaviour of Solutions to the Dirichlet Problem for Second Order Elliptic Equations near Edges and Polyhedral Vertices with Critical Angles
}

\author{
V. G. Maz'ya and J. Rossmann
}

\begin{abstract}
The Dirichlet problem for second order elliptic equations will be considered in domains of $\mathbb{R}^{N}$ with smooth $(N-2)$-dimensional edges at the boundary. The authors get the asymptotical decomposition of the solution near edges with angles running through a critical value. Furthermore, the first terms of the asymptotics of the solution near a polyhedral vertex are given for a domain with critical angle $\pi / 2$ in the vertex.
\end{abstract}

Keywords: Second order elliptic equations, asymptotics

AMS subject classification: $35 \mathrm{~J} 15$

\section{Introduction}

The present paper concerns the asymptotic behaviour of solutions of the Dirichlet problem for elliptic differential equations of second order in domains with edges if the angle at the edge runs through a critical value. It is known (see, e.g., $[3,4,7]$ ) that two different cases have to be considered in the description of the behaviour of the solution of the Dirichlet problem for the Laplacian near angular points: the resonance case where the asymptotics of the solution contains logarithmic terms and the non-resonance case without logarithmic terms. Logarithmic terms only occur if the angle at the corner is equal to a critical value. For the Laplacian such critical values may be all numbers of the form $j \pi / k$ where $j, k$ are integers. If we fix an integer number $l$ and consider the asymptotic decomposition

$$
u=\Sigma_{l}+u_{l}
$$

of the solution $u$ with a regular term $u_{l} \in W^{l+2+\varepsilon}(G)$, then all angles of the form

$$
\alpha^{*}=j \pi / k \quad(k=1,2, \ldots, l+1 ; j=1,2, \ldots, 2 k)
$$

become critical in the above sense. In the study of the asymptotics of the solution of the Dirichlet problem near edges one is confronted with difficulties if the angle on the edge varies and runs through a critical value.

V.G. Maz'ya: Linköping Univ., Dep. Math., S - 58183 Linkōping

J. Rossmann: Univ. Rostock, FB Math., Universitātspl. 1, D - 18051 Rostock 
Asymptotic decompositions for the solution of the Dirichlet problem for second order differential equations have been obtained by V.A. Kondratjev [5] and V.A. Nikishkin [14]. In several papers of V.G. Maz'ya and.B.A. Plamenevskij [6, 8], V.G. Maz'ya and J. Rossmann [9, 10], M. Dauge [3], J. Rossmann [17] these results have been generalized to boundary value problems for differential equations of higher order. Here critical angles have been either excluded or the authors considered only operators with constant coefficients in domains with constant angles at the edges. S. Rempel and B.-W. Schulze (see, e.g.; $[16,18,19]$ ) investigated pseudodifferential equations on manifolds with edges. They have given a very abstract description of the asymptotics of the solution by means of analytic functionals. The only condition in their papers is that the manifold is diffeomorphic to

$$
\Omega=\left\{x=\left(x^{\prime}, y\right) \in \mathbb{R}^{N}: y \in \mathbb{R}^{q}, x^{\prime} \in K\right\}
$$

in a neighbourhood of each edge point where $K$ denotes a cone in $\mathbb{R}^{N-q}$ which is independent of $y$. An explicite representation of the asymptotics of the solutions to boundary value problems for elliptic differential equations of second order has been first announced by M. Costabel and M. Dauge [1] (detailed proofs are given in [2]). Independently of them the authors of the present paper obtained a stable representation for the asymptotics of the solution of the Dirichlet problem in plane domains with angular points if the opening of the angle belongs to a neighbourhood of a critical value $\alpha^{*}$ (see [13]). In this case the singular functions

$$
r^{t \pi / \alpha+\mu} \psi(\phi)
$$

( $r, \phi$ denote the polar coordinates) in the asymptotics of the solution were replaced by more complicated singular functions

$$
z^{t \pi / \alpha+\mu} \bar{z}^{\nu} S_{\kappa}(z, \alpha)=z^{\mu+k_{n}+t \pi / \alpha} \bar{z}^{\nu} \cdot\left(\alpha-\alpha^{*}\right)^{-n} \sum_{j=0}^{n} a_{j} z^{k_{j}\left(\alpha^{*}-\alpha\right) / \alpha}
$$

$\left(a_{j}=-\sum_{\nu=1, \nu \neq j}^{n} k_{\nu} /\left(k_{\nu}-k_{j}\right), z=r e^{i \phi}\right)$ and conjugate terms.

The present paper is a direct continuation of [11] and [13]. In Section 3 we will show. that the solution of the Dirichlet problem for second order elliptic equations with smooth real coefficients near edges can be represented as a finite sum of singular functions

$$
c(x) \partial_{y}^{\beta}\left(z^{\imath \pi / \alpha+\mu} \bar{z}^{\nu} S_{\kappa}(z, \alpha)\right)
$$

and conjugate terms and a regular remainder (see Theorem 2). Along with the values $a^{*}=j \pi / k(k=1,2, \ldots, l+1 ; j=1,2, \ldots, 2 k-1 ; j \neq k)$ which are critical for the Laplacian we consider the case of the angle at the edge running through the value $\pi$ or $2 \pi$ (see Figure 1).

This case was excluded in $[1,2,11]$. The angles $\alpha^{*} \doteq \pi$ and $\alpha^{*}=2 \pi$ can be considered as critical too, because in a neighbourhood of such points the domain is not diffeomorphic to a dihedron $\mathcal{D}=\left\{x=\left(x_{1}, x_{2}, y\right): y \in \mathbb{R}^{N-2}, 0<\phi=\arg \left(x_{1}+i x_{2}\right)<\right.$ $\alpha$ ) with constant angle $\alpha$. In these cases it is necessary to consider the problem in a more general "dihedron"

$$
\mathcal{D}=\left\{x=\left(x_{1}, x_{2}, y\right): y \in \mathbb{R}^{N-2}, 0<\phi=\arg \left(x_{1}+i x_{2}\right)<\omega(r, y)\right\}
$$


$\left(r=\left(x_{1}^{2}+x_{2}^{2}\right)^{1 / 2}, \omega \in C^{\infty}\left(\overline{R_{+}} \times \mathbb{R}^{N-2}\right)\right)$ with angle $\alpha(y)=\omega(0, y)$ running through
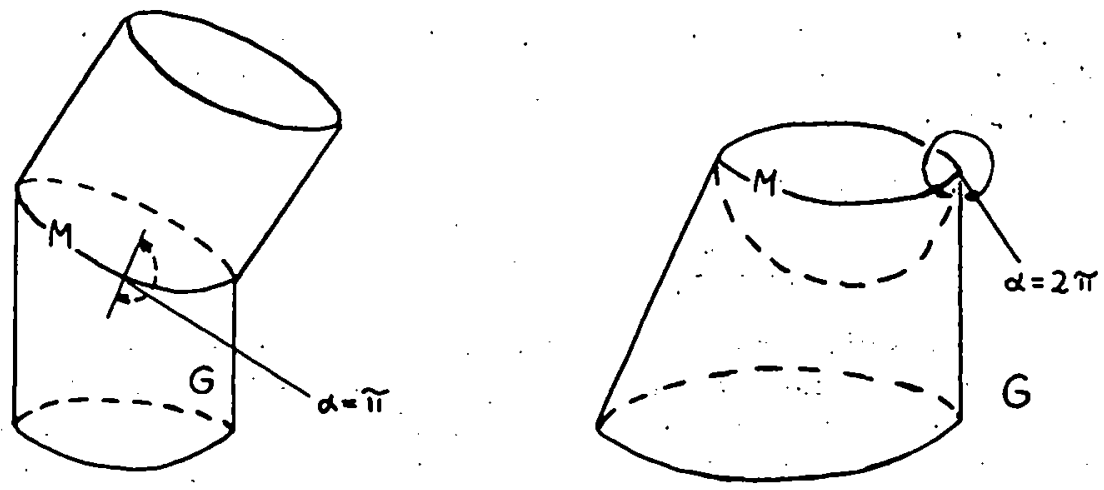

Fig. 1

$\pi$ or $2 \pi$. Note that in the special case when $\omega$ only depends on the variable $y$ the angles $\pi$ and $2 \pi$ are not critical. Then the solution $u$ admits the decomposition

$$
u=\sum c(x) r^{\mu+t \pi / \alpha(y)} \log ^{\nu} r \psi_{t, \mu, \nu}(\phi, y)+u_{1}
$$

$\left(u_{l} \in W^{l+2+e^{\prime}}\right.$ if $f \in W^{l+e} ; 0<\varepsilon^{\prime}<\varepsilon$ ) which is the same as for non-critical angles (cf. $[10,14])$.

In order to illustrate the representation of $u$ for other critical values $\alpha^{*}=j \pi / k(j / k$ non-integer) we consider the Dirichlet problem

$$
L u=f \quad \text { in } G, \quad u=0 \quad \text { on } \partial G \quad\left(f \in W^{i+\varepsilon}(G), \varepsilon>0\right)
$$

in a domain $G$ which coincides with the dihedron $D$ in a neighbourhood of a point $x_{0}$ on the edge $M$. Here we assume that the function $\omega$ in the definition of $\mathcal{D}$ does not depend on the variable $r$ and lies in a neighbourhood of $\alpha^{*}=\pi / 2$. Furthermore, we suppose that

$$
L=\sum_{\mu+\nu+|\beta| \leq 2} a_{\mu \nu \beta}(x) \partial_{x_{1}}^{\mu} \partial_{x_{2}}^{\nu} \partial_{y}^{\beta}
$$

is an elliptic differential operator with smooth real coefficients satisfying the condition

$$
a_{2,0,0}(0,0, y) \equiv a_{0,2,0}(0,0, y) \equiv 1, \quad a_{1,1,0}(0,0, y) \equiv 0
$$

For constant $\alpha$ the asymptotic decomposition of the solution $u \in \dot{W}^{1}(G)$ takes the form

$$
u=c_{1}(x) r^{\pi / \alpha} \sin \frac{\pi \phi}{\alpha}+c_{2}(x) r^{2}\left(\frac{1-\cos 2 \phi}{4}-\frac{1-\cos 2 \alpha}{4 \sin 2 \alpha} r^{2} \sin 2 \phi\right)+u_{1}
$$


if $\alpha \neq \pi / 2$ and

$$
u=c_{1}(x) r^{2} \sin 2 \phi+c_{2}(x) r^{2}\left(\frac{1-\cos 2 \phi}{4}+\frac{\phi}{\pi} \cos 2 \phi+\frac{1}{\pi} \log r \sin 2 \phi\right)+u_{1}
$$

if $\alpha=\pi / 2$ where $u_{1} \in W^{3+e}, r^{-3-e} u_{1} \in L_{2}$ and $c_{j}$ are functions from some weighted Sobolev spaces (see, e.g., [10]). By Theorem 2 of the present paper the solution $u$ admits the decomposition

$$
\begin{aligned}
u= & C_{1}(x) r^{\pi / \alpha(y)} \sin \frac{\pi \phi}{\alpha(y)} \\
& +C_{2}(x) \frac{1-\cos 2 \alpha(y)}{4 \sin 2 \alpha(y)}\left(r^{\pi / \alpha(y)} \sin \frac{\pi \phi}{\alpha(y)}-r^{2} \sin 2 \phi\right)+u_{1}
\end{aligned}
$$

if the angle $\alpha$ is variable und runs through the critical value $\alpha^{*}=\pi / 2$. Analogously to [9: Corollary 3.2 and Remark 3.3] and [10: Remark 4.1] the coefficients $C_{1}$ and $C_{2}$ can be replaced by their traces on the edge if $f$ belongs to the space $W^{*}$ where $s>2+\varepsilon+\sup \pi / \alpha(y)$. This corresponds to the so-called tensor product decomposition of T.v. Petersdorff and E.P. Stephan [15].

The results of Section 3 can be applied to the Dirichlet problem for second order elliptic equations in polyhedral domains if the angle at one of the edges is critical in a vertex. As an example we consider the Dirichlet problem

$$
L u=\sum_{\mu+\nu+k \leq 2} a_{\mu \nu k}(x) \partial_{x_{1}}^{\mu} \partial_{x_{2}}^{\nu} \partial_{x_{3}}^{k} u=f \text { in } G, \quad u=0 \text { on } \partial G
$$

in a domain $G$ which coincides with the infinite cube $(0, \infty)^{3}$ in a neighbourhood of the origin. If the principal part of $L$ with coefficients frozen in the origin is equal to the Laplacian, then the angle $\pi / 2$ in the origin is critical. It will be shown that the solution $u \in \dot{W}^{1}(G)$ admits the following decomposition in a neighbourhood of the $x_{3}$-axis if $f \in W^{1+e}(G)$ :

$$
\begin{aligned}
u= & \widehat{d}_{1}\left(x^{\prime}\right) r^{\prime \pi / \alpha\left(x_{3}\right)} \sin \left(\pi \phi / \alpha\left(x_{3}\right)\right) \\
& +\widehat{d}_{2}\left(x^{\prime}\right)\left(r^{\prime 2}\left(1-\cos 2 \phi^{\prime}\right)+\frac{\left(\alpha\left(x_{3}\right)-\pi / 2\right)\left(\cos \left(2 \alpha\left(x_{3}\right)\right)-1\right)}{\sin \left(2 \alpha\left(x_{3}\right)\right)}\right. \\
& \left.\times \frac{r^{\prime 2} \sin 2 \phi^{\prime}-x_{3}^{2-\pi / \alpha} r^{\prime \pi / \alpha} \sin \left(\pi \phi^{\prime} / \alpha\left(x_{3}\right)\right)}{\alpha\left(x_{3}\right)-\pi / 2}\right)+u^{\prime}
\end{aligned}
$$

Here $u^{\prime} \in W^{3}(G), r^{\prime-3} u \in L_{2}(G)$, the coordinates $x_{1}^{\prime}, x_{2}^{\prime}, x_{3}^{\prime}$ are defined by the equations

$$
\begin{aligned}
x_{1}^{\prime} & =\left(a_{0,2,0}\left(0,0, x_{3}\right) / D\right)^{1 / 2} x_{1}-\frac{1}{2} a_{1,1,0}\left(0,0, x_{3}\right)\left(a_{0,2,0}\left(0,0, x_{3}\right) D\right)^{-1 / 2} x_{2} \\
x_{2}^{\prime} & =a_{0,2,0}\left(0,0, x_{3}\right)^{-1 / 2} x_{2} \\
x_{3}^{\prime} & =x_{3}
\end{aligned}
$$

where

$$
\begin{aligned}
& D=a_{2,0,0}\left(0,0, x_{3}\right) a_{0,2,0}\left(0,0, x_{3}\right)-\frac{1}{4} a_{1,1,0}\left(0,0, x_{3}\right) \\
& r^{\prime}=\left(x_{1}^{\prime 2}+x_{2}^{\prime 2}\right)^{1 / 2}, \varphi^{\prime}=\arg \left(x_{1}^{\prime}+i x_{2}^{\prime}\right), \tan \alpha\left(x_{3}\right)=-2 D^{1 / 2} / a_{1,1,0}\left(0,0, x_{3}\right)
\end{aligned}
$$

and $\hat{d}_{1}, \hat{d}_{2}$ are some extensions of functions on the $x_{3}$-axis.

The authors are grateful to M. Costabel and M. Dauge for useful remarks in connection with this paper. 


\section{Regularity of the derivatives of the solution in edge direction}

Let $\mathcal{D}$ be the dihedron

$$
\mathcal{D}=\left\{x=\left(x_{1}, x_{2}, y\right) \in \mathbb{R}^{N}: y \in \mathbb{R}^{N-2}, 0<\phi<\omega(r, y)\right\}
$$

with variable angle $\alpha(y)=\omega(0, y)$ (see Figure 2). Here $r=\left(x_{1}^{2}+x_{2}^{2}\right)^{1 / 2}, \phi=\arg \left(x_{1}+\right.$ $\left.i x_{2}\right)$ are the polar coordinates in the $\left(x_{1}, x_{2}\right)$-plane and $\omega$ is an infinitely differentiable

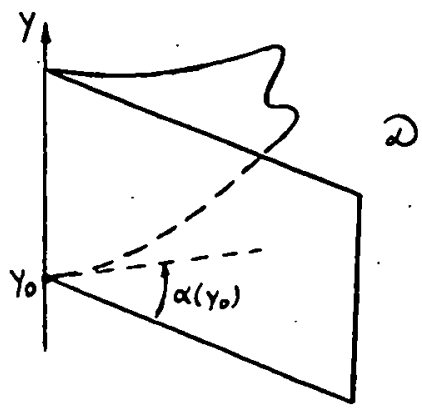

Fig. 2

function on $\bar{R}_{+} \times \mathbb{R}^{N-2}$ such that $0<\inf _{r, y} \omega(r, y) \leq \sup _{r, y} \omega(r, y) \leq 2 \pi$. The edge $M$ of $\mathcal{D}$ coincides with the $y$-axis. We consider the Dirichlet problem

$$
L u=\sum_{\mu+\nu+|\beta| \leq 2} a_{\mu, \nu \beta}\left(x_{1}, x_{2}, y\right) \partial_{x_{1}}^{\mu} \partial_{x_{2}}^{\nu} \partial^{\beta} u=f \text { in } \mathcal{D}, \quad u=0 \text { on } \partial \mathcal{D}
$$

Here $L$ is an arbitrary elliptic differential operator of second order with real coefficients $a_{\mu, \nu, \beta} \in C^{\infty}(\overline{\mathcal{D}})$. Without loss of generality we may assume that

$$
a_{2,0,0}(0,0, y) \equiv a_{0,2,0}(0,0, y) \equiv 1, \quad a_{1,1,0}(0,0, y) \equiv 0 .
$$

Otherwise we make use of the diffeomorhpism

$$
\begin{aligned}
x_{1}^{\prime} & =\left(a_{0,2,0}(0,0, y) / D\right)^{1 / 2} x_{1}-\frac{1}{2} a_{1,1,0}(0,0, y)\left(a_{0,2,0}(0,0, y) D\right)^{-1 / 2} x_{2} \\
x_{2}^{\prime} & =a_{0,2,0}(0,0, y)^{-1 / 2} x_{2} \\
y^{\prime} & =y
\end{aligned}
$$

where $D=a_{2,0,0}(0,0, y) a_{0,2,0}(0,0, y)-\frac{1}{4} a_{1,1,0}(0,0, y)^{2}$. We introduce the following weighted Sobolev spaces. By $V_{\delta}^{\prime}(\mathcal{D})\left(l\right.$ integer, $l \geq 0, \delta \in C^{\infty}\left(\mathbb{R}^{N-2}\right)$ real-valued) we denote the closure of $C_{0}^{\infty}(\overline{\mathcal{D}} \backslash M)$ with respect to the norm

$$
\|u\|_{V_{j}^{\prime}(\mathcal{D})}=\left(\int_{\mathcal{D}} \sum_{|\beta| \leq l} r^{2(\delta(y)-1+|\beta|)}\left|D^{\beta} u\right|^{2} d x\right)^{1 / 2}
$$


Furthermore, the space $W_{\delta}^{l}(\mathcal{D})\left(l\right.$ integer, $\left.l \geq 0, \delta \in C^{\infty}\left(\mathbb{R}^{N-2}\right), \delta>-1\right)$ will be defined as the closure of $C_{0}^{\infty}(\overline{\mathcal{D}})$ with respect to the norm

$$
\|u\|_{W_{\jmath}^{\prime}(\mathcal{D})}=\left(\int_{\mathcal{D}} \sum_{|\beta| \leq l} r^{2 \delta(y)}\left|D^{\beta} u\right|^{2} d x\right)^{1 / 2}
$$

Note that the usual Sobolev-Slobodezkij space $W^{l+e}(\mathcal{D})$ is continuously imbedded into $W_{-\varepsilon}^{\prime}(\mathcal{D})$ if $\varepsilon$ is a real number from the interval $(0,1)$. Analogously to [8] the following lemma can be proved.

Lemma 1. Let $u \in V_{-\varepsilon}^{1}(\mathcal{D})$ be a solution of problem (1.2) with $f \in V_{l+1-\varepsilon}^{l}(\mathcal{D})$ $(l \geq 0$ integer, $\varepsilon \in \mathbb{R})$. Suppose that the support of $u$ is compact. Then $u \in V_{l+1-e}^{l+2}(\mathcal{D})$ and

$$
\|u\|_{V_{i+1-\varepsilon}^{\prime+2}(D)} \leq c\left(\|u\|_{V_{-,}^{1}(D)}+\|f\|_{V_{i+1-\varepsilon}^{\prime}(D)}\right) .
$$

Here the constant $c$ depends only on $\mathcal{D}$ and the support of $u$.

Proof. We only sketch the proof. Let $u=0$ for $|x|>R$. There exists a countable collection of open balls $B_{j}$ which cover $\mathcal{D} \cap\{x:|x|>R\}$ and satisfy the conditition $d_{j}=\operatorname{diam} B_{j}=\operatorname{dist}\left(B_{j}, M\right)$. Furthermore, let $B_{j}^{\prime}$ be balls concentric to $B_{j}$ with diameter $2 d_{j}$. From the classical $L_{2}$-estimates for solutions of elliptic equations in domains with smooth boundaries it follows

$$
\begin{aligned}
& \int_{B_{j} \cap \mathcal{D}} \sum_{|\beta| \leq l+2} d_{j}^{2|\beta|}\left|D^{\beta} u\right|^{2} d x \\
& \leq c \int_{B_{j}^{\prime} \cap \mathcal{D}}\left(\sum_{|\beta| \leq 1} d_{j}^{2|\beta|}\left|D^{\beta} u\right|^{2}+\sum_{|\beta| \leq l} d_{j}^{2|\beta|+4}\left|D^{\beta} f\right|^{2}\right) d x .
\end{aligned}
$$

Multiplying this inequality by $d_{j}^{-2 e-2}$ and summing up over all $j$ we get (1.4)

If $f$ is an arbitrary function in $\mathcal{D}$ (given in cylindrical coordinates $r, \phi, y$ ) and $h \in \mathbb{R}$, then we denote by $f_{h}$ the function

$$
\begin{aligned}
& f_{h}(r, \phi, y)= \\
& \quad \frac{1}{h}\left(f\left(r, \frac{w\left(r, y_{1}+h, y_{2}, \ldots, y_{N-2}\right)}{\omega(r, y)} \phi, y_{1}+h, y_{2}, \ldots y_{N-2}\right)-f(r, \phi, y)\right) .
\end{aligned}
$$

Obviously,

$$
\lim _{h \rightarrow 0} f_{h}(r, \phi, y)=\partial_{y_{1}} f(r, \phi, y)+\frac{\phi}{\omega(r, y)}\left(\partial_{y_{1}} \omega(r, y)\right) \partial_{\phi} f(r, \phi, y)
$$

Note that the derivative

$$
D_{j}=\partial_{y_{j}}+\frac{\phi}{\omega(r, y)}\left(\partial_{y_{j}} \omega(r, y)\right) \partial_{\phi}
$$


$(j=1, \ldots, N-2)$ is tangential on the sides $\phi=0$ and $\phi=\omega(r, y)$ of $\mathcal{D}$. We furthermore mention the following property of the operator $D_{j}$ : if $\mathcal{L}$ is an arbitrary second order differential operator of the form.

$$
\mathcal{L}=\sum_{\mu+\nu+|\beta| \leq 2} a_{\mu \nu \beta}(r, \phi, y) r^{-\nu} \partial_{r}^{\mu} \partial_{\phi}^{\nu} \partial_{y}^{\beta}
$$

with coefficients $a_{\mu \nu \beta}$ being infinitely differentiable relative to $r, \phi, y$, then there exists an operator $\mathcal{L}_{\text {; }}$ of the form (1.6) such that

$$
D_{j} \mathcal{L} u=\mathcal{L}\left(D_{j} u\right)+\mathcal{L}_{j} u .
$$

Lemma 2. Let $u \in V_{-e}^{1}(\mathcal{D}), f \in V_{-e}^{0}(\mathcal{D}) \quad(\varepsilon \in \mathbb{R})$ be arbitrary functions on $\mathcal{D}$ such that $u(x)=0, f(x)=0$ for $r>R$. Then the following inequalities hold with constants $c_{1}, c_{2}, c_{3}$ independent of $h, u$ and $f$ :

$$
\begin{aligned}
& \left\|u_{h}\right\|_{V_{-}^{\circ}(\mathcal{D})} \leq c_{2}\left\|D_{1} u\right\|_{V_{-.}^{0}(D)} \leq c_{2}\|u\|_{V_{-}^{1}(\mathcal{D})} \\
& \left\|f_{h}\right\|_{V_{-!}^{-1}(D)} \leq c_{3}\|f\|_{V_{-}^{0}(D)}
\end{aligned}
$$

where $V_{-z}^{-1}(\mathcal{D})$ denotes the dual space of $V_{e}^{1}(\mathcal{D})$.

Proof. For simplicity we restrict ourselves to the case $N=3$. For $N>3$ the lemma can be proved analogously.

a) Using the equation

$$
\begin{aligned}
u_{h}= & \frac{1}{h} \int_{y}^{y+h} \frac{d}{d s} u\left(r, \frac{\omega(r, s)}{\omega(r, y)} \phi, \dot{s}\right) d s \\
= & \int_{0}^{1}\left(\frac{\partial u}{\partial y}\left(r, \frac{\omega(r, y+t h) \phi}{\omega(r, y)}, y+t h\right)\right. \\
& \left.+\phi \frac{\partial_{y} \omega(r, y+t h)}{\omega(r, y)} \frac{\partial u}{\partial \phi}\left(r, \frac{\omega(r, y+t h) \phi}{\omega(r, y)}, y+t h\right)\right) d t
\end{aligned}
$$

we get

$$
\begin{aligned}
& \int_{\mathcal{D}} r^{-2 e}\left|u_{h}\right|^{2} d x \leq \int_{0}^{1} \int_{0}^{\infty} \int_{\mathbb{R}}^{\infty} \int_{0}^{\omega(r, y)} r^{1-2 e} \mid \frac{\partial u}{\partial y}\left(r, \frac{\omega(r, y+t h)}{\omega(r, y)} \phi, y+t h\right) \\
& +\left.\phi \frac{\partial_{y} \omega(r, y+t h)}{\omega(r, y)} \frac{\partial u}{\partial \phi}\left(r, \frac{\omega(r, y+t h)}{\omega(r, y)} \phi, y+t h\right)\right|^{2} d \phi d y d r d t \\
& \leq \int_{0}^{\infty} \int_{\mathbb{R}}^{\omega(r, y)} \int_{0}^{1-2 e}\left|D_{1} u\right|^{2} d \phi d y d r \\
& =c \int_{\mathcal{D}} r^{-2 e}\left|D_{1} u\right|^{2} d x \leq c\|u\|_{V_{-\cdot}^{2}(D)}^{2}
\end{aligned}
$$


b) It can be easily verified that

$$
\int_{\mathcal{D}} f_{h} v d x=-\int_{\mathcal{D}} f\left(\frac{\omega(r, y-h)}{\omega(r, y)} v_{-h}+\frac{1}{\omega(r, y)} \frac{\dot{\omega}(r, y)-\omega(r, y-h)}{h} v\right) d x .
$$

Consequently,

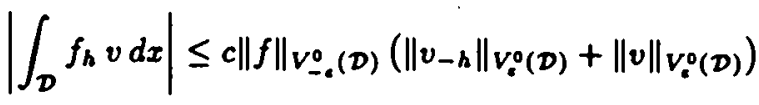

$$
\begin{aligned}
& \leq c\|f\|_{V_{-e}^{0}(\mathcal{D})}\|v\|_{V_{e}^{1}(\mathcal{D})} \text {. }
\end{aligned}
$$

The result follows

In a similar way one can prove the following lemma.

Lemma 3. Let $u \in V_{\delta}^{2}(\mathcal{D})(\delta \in \mathbb{R})$ be an arbitrary function satisfying the condition $u \equiv 0$ for $r>R$. Then the estimate

$$
\left\|(L u)_{h}-L_{u_{h}}\right\|_{\left.V_{j}^{(D}\right)} \leq c\|u\|_{V_{f}^{2}(\mathcal{D})}
$$

is valid with a constant $c$ independent of $u$ and $h$.

Now we can prove the following regularity assertion for the derivatives $\partial u / \partial y_{j}(j=$ $1,2, \ldots, N-2)$. For constant $\omega$ this assertion has been shown in $[5,9,10]$. We use the same technique.

Proposition 1. Let $u \in \dot{W}^{1}(\mathcal{D})$ be a solution of problem (1.2) where $f=f_{1}+$ $f_{2}$ with $f_{1} \in V_{-\varepsilon}^{0}(\mathcal{D}), f_{2} \in V_{-\varepsilon+1}^{0}(\mathcal{D})$ and $D_{j} f_{2} \in V_{-\varepsilon+1}^{0}(\mathcal{D})(j=1, \ldots, N-2 ; \varepsilon \geq$ $0, \varepsilon$ sufficiently small). Moreover, we assume that $u \equiv 0$ for $r>R$. Then $u \in$ $V_{-e}^{1}(\mathcal{D}), \partial u / \partial y_{j} \in V_{-e}^{1}(\mathcal{D})$ and

$$
\|u\|_{V_{-}^{1}(\mathcal{D})}+\sum_{j=1}^{N-2}\left\|\frac{\partial u}{\partial y_{j}}\right\|_{V_{-,}^{1}(\mathcal{D})} \leq c\left(\|u\| W^{1}(\mathcal{D})+\|f\|\right)
$$

with a constant $c$ independent of $u$ and $f$. Bere $\|\cdot\|$ denotes the norm

$$
\|f\|=\inf _{f=f_{1}+f_{2} \cdot}\left\{\left\|f_{1}\right\|_{V_{-}^{0}(\mathcal{D})}+\left\|f_{2}\right\|_{V_{-\bullet}^{0}(\mathcal{D})}+\sum_{j=1}^{N-2}\left\|D_{j} f_{2}\right\|_{V_{-}^{0},(D)}\right\} .
$$

Proof. At first we show that every solution $u \in V_{-e}^{1}(\mathcal{D})$ of problem (1.2) with $f$ as in the formulation of the proposition satisfies the inequality

$$
\left\|\partial_{y_{j}} u\right\|_{V_{-\bullet}^{1}(\mathcal{D})} \leq c\left(\|u\|_{V_{-\bullet}^{1}(\mathcal{D})}+\|f\|\right) .
$$

Since $f \in V_{-e+1}^{0}(\mathcal{D})$ Lemma 1 implies $u \in V_{-e+1}^{2}(\mathcal{D})$ and

$$
\|u\|_{V_{-c+1}^{2}(D)} \leq c\left(\|u\|_{V_{-\bullet}^{1}(\mathcal{D})}+\|f\|_{V_{-c+1}^{0}(D)}\right) \leq c\left(\|u\|_{V_{-\bullet}^{1}(\mathcal{D})}+\|f\|\right) .
$$


Furthermore, $r^{-e} u_{k} \in W^{1}(\mathcal{D})$. Then the Gärding inequality yields

$$
\begin{aligned}
\left\|r^{-\varepsilon} u_{h}\right\|_{V_{0}^{1}(\mathcal{D})}^{2} & \leq c\left\|r^{-\varepsilon} u_{h}\right\|_{W^{1}(\mathcal{D})}^{2} \\
& \leq c\left(\left|\left(L\left(r^{-\varepsilon} u_{h}\right), r^{-\varepsilon} u_{h}\right)\right|+\left\|r^{-\varepsilon} u_{h}\right\|_{L_{2}(\mathcal{D})}^{2}\right),
\end{aligned}
$$

i. e.

$$
\begin{aligned}
\left\|r^{-\varepsilon} u_{h}\right\|_{V_{0}^{1}(\mathcal{D})} & \leq c\left(\left\|L\left(r^{-\varepsilon} u_{h}\right)\right\|_{V_{0}^{-1}(\mathcal{D})}+\left\|r^{-\varepsilon} u_{h}\right\|_{L_{2}(\mathcal{D})}\right) \\
& \leq c\left(\left\|\left[L, r^{-\varepsilon}\right] u_{h}\right\|_{V_{0}^{-1}(\mathcal{D})}+\left\|r^{-\varepsilon} L u_{h}\right\|_{V_{0}^{-1}(\mathcal{D})}+\left\|r^{-\varepsilon} u_{h}\right\|_{L_{2}(\mathcal{D})}\right)
\end{aligned}
$$

$\left(\left[L, r^{-\varepsilon}\right]\right.$ denotes the commutator of $L$ and $\left.r^{-e}\right)$. By Lemma 2 and Lemma 3 we have

$$
\begin{aligned}
& \left\|r^{-\ell} L u_{h}\right\|_{V_{0}^{-1}(\mathcal{D})} \\
& \leq c\left(\| r^{-e}\left(L u_{h}-(L u)_{h}\left\|_{V_{0}^{-1}(\mathcal{D})}+\right\| f_{h} \|_{V_{-\ell}^{-1}(\mathcal{D})}\right)\right. \\
& \leq c\left(\left\|L u_{h}-(L u)_{h}\right\|_{V_{-e+1}^{0}(\mathcal{D})}+\left\|\left(f_{1}\right)_{h}\right\|_{V_{-:}^{-1}(\mathcal{D})}+\left\|\left(f_{2}\right)_{h}\right\|_{V_{-S+1}^{0}(\mathcal{D})}\right) \\
& \leq c\left(\|u\|_{V_{-\varepsilon+1}^{2}(\mathcal{D})}+\left\|f_{1}\right\|_{V_{-\varepsilon}^{0}(\mathcal{D})}+\left\|D_{1} f_{2}\right\|_{V_{-\ell+1}^{0}(\mathcal{D})}\right)
\end{aligned}
$$

for an arbitrary decomposition $f=f_{1}+f_{2}$ of the function $f$ where $f_{1} \in V_{-2}^{0}(\mathcal{D}), f_{2} \in$ $V_{-\varepsilon+1}^{0}(\mathcal{D})$ and $D_{1} f_{2} \in V_{\varepsilon+1}^{0}(\mathcal{D})$. Here we have used the continuity of the imbedding $V_{-e+1}^{0}(\mathcal{D}) \subset V_{-\varepsilon}^{-1}(\mathcal{D})$. Furthermore,

$$
\left\|\left[L, r^{-\varepsilon}\right] u_{h}\right\|_{V_{0}^{-1}(\mathcal{D})} \leq c \varepsilon\left\|r^{-\varepsilon} u_{h}\right\|_{V_{0}^{1}(\mathcal{D})}
$$

and

$$
\left\|r^{-\varepsilon} u_{h}\right\|_{L_{2}(\mathcal{D})}=\left\|u_{h}\right\|_{V_{-}^{0}(\mathcal{D})} \leq c\|u\|_{V_{-}^{1}(\mathcal{D})}
$$

with a constant $c$ independent of $\varepsilon$ and $h$. Consequently, for sufficiently small $\varepsilon$ we get

$$
\left\|u_{h}\right\|_{V_{-\bullet}^{1}(\mathcal{D})} \leq c\left(\|u\|_{V_{-\cdot+1}^{2}(\mathcal{D})}+\left\|f_{1}\right\|_{V_{-e}^{0}(\mathcal{D})}+\left\|D_{1} f_{2}\right\|_{V_{-\cdot+1}^{0}(\mathcal{D})}\right)
$$

i.e.

$$
\left\|\frac{\partial u}{\partial y_{1}}\right\|_{V_{-\bullet}^{1}(\mathcal{D})} \leq c\left(\|u\|_{V_{-\bullet}(\mathcal{D})}+\|f\|\right)
$$

Analogously one gets the same estimate for the derivatives $\partial u / \partial y_{j}(j=2, \ldots, N-2)$. According to the assumption of the proposition $u$ belongs to $\dot{W}^{1}(\mathcal{D}) \subset V_{0}^{1}(\mathcal{D})$. Hence, by (1.8), $\partial_{y_{j}} u \in V_{0}^{1}(\mathcal{D})$. Then from [4] it follows that $u \in V_{-e}^{1}(\mathcal{D})$ for sufficiently small $\varepsilon>0$ (see also [5]) and $\|u\|_{V_{-,}^{1}(\mathcal{D})} \leq c\left(\|u\|_{V_{0}^{1}(\mathcal{D})}+\|f\|_{V_{-}^{0},(\mathcal{D})}\right)$. Applying again (1.8) one gets the assertion of the proposition 
Theorem 1. Let $u \in W^{1}(\mathcal{D})$ be a solution of problem (1.2) with compact support where $D_{y}^{\beta} f \in V_{-e+k-|\beta|}^{k-|\beta|}(\mathcal{D})$ for $|\beta| \leq k$ and $\varepsilon$ is a sufficiently small non-negative real number (this condition on the function $f$ is satisfied, e.g., if $f \in W_{-e}^{k}(\mathcal{D})$ ). Then $D_{y}^{\beta} u \in V_{-e+k+1-|\beta|}^{k+2-|\beta|}(\mathcal{D}) \subset V_{-e}^{1}(\mathcal{D})$ for $|\beta| \leq k+1$.

Proof. This generalization of Proposition 1 can be easily shown by induction in $k$. Assume that $N=3$ and the assertion is true for $k-1$. Then we get $\partial_{y}^{j} u \in V_{-e+k-j}^{k+1-j}(\mathcal{D})$ for $j=0,1, \ldots, k$, i.e. $D_{1}^{j} u \in V_{-\varepsilon+k-j}^{k+1-j}(\mathcal{D})$ for $j=0,1, \ldots, k$. From (1.7) it follows that $L\left(D_{1}^{k} u\right)$ can be written in the form

$$
L\left(D_{1}^{k} u\right)=D_{1}^{k}(L u)+\sum_{j=0}^{k-1} L_{j}\left(D_{1}^{j} u\right)=D_{1}^{k} f+\sum_{j=0}^{k-1} L_{j}\left(D_{1}^{j} u\right)
$$

where $L_{j}$ are second order differential operators of the form (1.6). Here $D_{1}^{k} f \in V_{-\varepsilon}^{0}(\mathcal{D})$ and $\sum L_{j}\left(D_{1}^{j} u\right) \in V_{-\varepsilon}^{0}(\mathcal{D})$ (since $D_{1}^{j} u \in V_{-\varepsilon+1}^{2}(\mathcal{D})$ for $j=0,1, \ldots, k-1$ ). Lemma 1 implies $D_{1}^{k} u \in V_{-e+1}^{2}(\mathcal{D})$. Furthermore, by (1.7) we have

$$
D_{1} \sum_{j=0}^{k-1} L_{j}\left(D_{1}^{j} u\right)=\sum_{j=0}^{k} \widetilde{L_{j}}\left(D_{1}^{j} u\right)
$$

where $\widetilde{L_{j}}$ are differential operators of the form (1.6). Hence $D_{1} \sum L_{j}\left(D_{1}^{j} u\right) \in V_{-e+1}^{0}(\mathcal{D})$ and by Proposition 1 we get $D_{1}^{k} u \in V_{-e}^{1}(\mathcal{D})$, i.e. the assertion of the theorem is true for every $k$. In the case $N>3$ the theorem can be proved analogously

\section{Solutions of the Dirichlet problem for special right-hand sides}

We introduce here the same singular functions as in [13]. Let $l$ be a given non-negative integer. An angle $\alpha^{*}$ is said to be critical if there exists an integer $k \in\{1,2, \ldots, l+1\}$ such that $k \alpha^{*} / \pi$ is integer. By $\mathcal{K}_{l}\left(\alpha^{*}\right)$ we denote the set of all tuples $\kappa=\left(k_{0}, k_{1}, \ldots, k_{n}\right)$ of integer numbers $k_{j}$ where $n \geq 0,0=k_{0}<k_{1}<\ldots<k_{n} \leq l+1$ and $k_{j} \alpha^{*} / \pi$ are integers. For a given tuple $\kappa=\left(k_{0}, k_{1}, \ldots, k_{n}\right) \in \mathcal{K}_{l}\left(\alpha^{*}\right)$ we define the function $S_{k}(z, \alpha)$ of the complex variable $z=x_{1}+i x_{2}=r e^{i \phi}(0 \leq \phi<2 \pi)$ and the angle $\alpha \in(0,2 \pi]$ as follows:

$$
S_{k}(z, \alpha)= \begin{cases}\left(\alpha-\alpha^{*}\right)^{-n} z^{k_{n}} \sum_{j=0}^{n} a_{j} z^{k_{j}\left(\alpha^{*}-\alpha\right) / \alpha} & \text { if } \alpha \neq \alpha^{*} \\ \lim _{\alpha \rightarrow \alpha^{*}}\left(\alpha-\alpha^{*}\right)^{-n} z^{k_{n}} \sum_{j=0}^{n} a_{j} z^{k_{j}\left(\alpha^{*}-\alpha\right) / \alpha} & \text { if } \alpha=\alpha^{*}\end{cases}
$$

where $a_{j}=-\prod_{\nu=1, \nu \neq j}^{n} k_{\nu} /\left(k_{\nu}-k_{j}\right)$ for $j=1, \ldots, n$ and $a_{0}=1$. In the case $n=0$ (i.e. $\kappa=\left(k_{0}\right)=(0)$ ) we set $S_{\kappa}(z, \alpha)=1$. It is shown in [13] that $S_{\kappa}(z, \alpha)$ is infinitely differentiable.relative to $\alpha$ for every $z \neq 0$. The limit

$$
\lim _{\alpha \rightarrow \alpha^{+}}\left(\alpha-\alpha^{*}\right)^{-n} z^{k_{n}} \sum_{j=0}^{n} a_{j} z^{k_{j}\left(\alpha^{*}-\alpha\right) / \alpha}
$$


is a polynomial in $\log z=\log r+i \phi$ of order $n$. If $\alpha$ is an infinitely differentisble function of the variable $y \in \mathbb{R}^{N-2}$, then $S_{\kappa}(z, \alpha(y))$ is infinitely differentiable relative to $y$; too.

We consider the problem (1.2) in the dihedron (1.1) where the angle $\alpha(y)$ of $\mathcal{D}$ lies in a neighbourhood of the critical angle $\alpha^{*}$. In the case $\alpha^{*} \neq \pi, \alpha^{*} \neq 2 \pi$ we can assume without loss of generality that the function $\omega$ in (1.1) does not depend on the variable $r$. The cases $\alpha^{*}=\pi$ and $\alpha^{*}=2 \pi$ we will consider separately.

a) The case $\alpha^{*} \neq \pi, 2 \pi$. It is useful to write the Laplace operator in the $\left(x_{1}, x_{2}\right)$-plane in the form

$$
\partial_{x_{1}}^{2}+\partial_{x_{2}}^{2}=4 \partial_{z} \partial \bar{z}
$$

where $\partial_{z}=\frac{1}{2}\left(\partial_{z_{1}}-i \partial_{z_{2}}\right)$ and $\partial_{\bar{z}}=\frac{1}{2}\left(\partial_{x_{1}}+i \partial_{x_{2}}\right)$. The following lemma has been proved in [13: Lemma 6].

Lemma 4. Let $\alpha^{*}$ be a critical angle and $\kappa=\left(k_{0} ; k_{1}, \ldots, k_{n}\right)$ a tuple from $\mathcal{K}_{l}\left(\alpha^{*}\right)$. Assume that $\alpha$ lies in a sufficiently small neighbourhood $U\left(\alpha^{*}\right)$ of $\alpha^{*}$. Then the problem

$$
\begin{aligned}
\partial_{z} \partial_{\bar{z}} v & =\partial_{z}^{q}\left\{z^{\mu+t \pi / \alpha} \bar{z}^{\nu} S_{\kappa}(z, \alpha)\right\} & & \text { for } 0<\arg z<\alpha \\
v & =0 & & \text { for } \arg z=0, \arg z=\alpha
\end{aligned}
$$

with non-negative integers $q, t, \mu, \nu, \mu+\nu \geq q-1$, has a solution of the form

$$
\begin{aligned}
v= & \sum_{j=0}^{n} A_{j}(\alpha) z^{\mu+1-q+k_{n}-k_{j}+t \pi / \alpha} \bar{z}^{\nu+1} S_{\kappa_{j}}(z, \alpha) \\
& +\sum_{j=0}^{n} B_{j}(\alpha) \operatorname{Re}\left\{z^{k_{n+1}-k_{j}+t \pi / \alpha} S_{\kappa_{j}}(z, \alpha)\right\} \\
& +\sum_{j=0}^{n+1} C_{j}(\alpha) \operatorname{Im}\left\{z^{k_{n+1}-k_{j}+t \pi / \alpha} S_{\kappa_{j}}(z, \alpha)\right\}
\end{aligned}
$$

where $k_{n+1}=k_{n}+\mu+\nu+2-q, \kappa_{j}=\left(k_{0}, k_{1}, \ldots, k_{j}\right)$ and $A_{j}, B_{j}, C_{j}$ are infinitely differentiable functions in $U\left(\alpha^{*}\right)$. If $q \geq 1$, then the first term in $(2.2)$ can be replaced by

$$
\frac{1}{\nu+1} \partial_{z}^{q-1}\left\{z^{\mu+t \pi / \alpha} \bar{z}^{\nu+1} S_{\kappa}(z, \alpha)\right\} \text {. }
$$

Furthermore, $C_{n+1} \equiv 0$ if $k_{n+1} \alpha^{*} / \pi$ is not an integer.

Remark 1. In Lemma 4 the cases $\alpha^{*}=\pi$ and $\alpha^{*}=2 \pi$ are included. In these cases the coefficient $C_{n+1}$ is identically equal to zero in the neighbourhood $U\left(\alpha^{*}\right)$ of $\alpha^{*}$ (see [13]).

In order to get a similar result for the Dirichlet problem (1.2) in the dihedron

$$
\mathcal{D}=\left\{x=\left(x_{1}, x_{2}, y\right): y \in \mathbb{R}^{N-2}, 0<\phi=\arg \left(x_{1}+i x_{2}\right)<\alpha(y)\right\}
$$

we need the following assertions on weighted Sobolev spaces. The proof is given, e.g., in $[9,10]$. 
Lemma 5. The following assertions are valid.

a) Let $v \in W_{\delta}^{\prime}(\mathcal{D})$ with a smooth function $\delta$ satisfying the condition $-1<$ inf $\delta \leq$ sup $\delta<l-1$ and $l \geq 1$. Then the trace of $v$ on $M$ lies in the Sobolev space $W^{l-1-\delta}(M)$.

b) Let $f \in W^{l-1-\delta}(M)(l \geq 1,-1<\inf \delta \geq \sup \delta<l-1)$. Then the function

$$
v(x)=(\mathcal{K} f)(x)=\chi(r) \int_{\mathbb{R}^{N-2}} f(z+t r) \psi\left(t_{1}\right) \cdots \psi\left(t_{N-2}\right) d t
$$

$\left(t=\left(t_{1}, \ldots, t_{N-2}\right), \chi \in C_{0}^{\infty}\left(\bar{R}_{+}\right)\right.$, supp $\chi \subseteq[0,1], \chi \equiv 1$ in $\left[0, \frac{1}{2}\right], \psi \in C_{0}^{\infty}(\mathbb{R})$, $\operatorname{supp} \psi \subseteq(-1,+1), \int_{R} s^{j} \psi(s) d s=\delta_{j, 0}$ for $\left.j=0,1, \ldots, l-1, r=\left(x_{1}^{2}+x_{2}^{2}\right)^{1 / 2}\right)$ is an extension of $f$ and belongs to the space $W_{\delta}^{l}(\mathcal{D})$. The extension operator (2.S) is a continuous mapping from $W^{l-1-\delta}(M)$ into $W_{b+\nu}^{l+\nu}(D)$ for every $\nu \geq 0$.

c) If $f \in W^{l-1-\delta}(M) \quad(l \geq 1,-1<$ inf $\delta \leq \sup \delta<0)$, then

(i) $\partial_{x_{1}}^{i} \partial_{x_{2}}^{j} \mathcal{K} f \in V_{\delta+\nu}^{l+\nu-i-j}(\mathcal{D})$ for each $i, j, \nu, 1 \leq i+j \leq l+\nu$

(ii) $D_{y}^{\beta} \mathcal{K} f \in V_{-1+\varepsilon}^{0}(\mathcal{D})$ for $|\beta| \leq l-1$ where $\varepsilon$ is an arbitrary positive number

(iii) $D_{y}^{\beta} \mathcal{K} f=\mathcal{K}\left(D_{y}^{\beta} f\right)$ for $|\beta| \leq l-1$

(iv) $c \mathcal{K} f-\mathcal{K}(c f) \in V_{\delta+\nu}^{l+\nu}(\mathcal{D})$ for every $c \in C^{\infty}(M),|\beta| \leq l-1$. sition

d) Every function $u \in W_{\delta}^{\prime}(\mathcal{D})(l \geq 1,-1<\inf \delta \leq \sup \delta<0)$ admits the decompo-

$$
u=P_{l}(u)+v
$$

where

$$
P_{l}(u)=\sum_{\mu+\nu \leq l-1} \frac{1}{\mu ! \nu !}\left(\mathcal{K}_{u_{\mu \nu}}\right) x_{1}^{\mu} x_{2}^{\nu}, \quad u_{\mu \nu}=\left.\partial_{x_{1}}^{\mu} \partial_{x_{2}}^{\nu} u\right|_{M}, \quad v \in V_{\delta}^{l}(\mathcal{D}) .
$$

We now can formulate the analogon to Lemma 4 for the Dirichlet problem (1.2). It corresponds to [10: Lemma 5.1].

Proposition 2. Let $\kappa=\left(k_{0}, k_{1}, \ldots, k_{n}\right)$ be a tuple from $\mathcal{K}_{l-1}\left(\alpha^{*}\right)$ and

$$
f=a(y) \widehat{c}(x) \partial_{y}^{\gamma} \partial_{z}^{q}\left\{z^{\mu+t \pi / \alpha(y)} \bar{z}^{\nu} S_{\kappa}(z, \alpha(y))\right\}
$$

where $1 \leq q \leq \mu+\nu+1, l-2+\varepsilon<\mu+\nu+k_{n}-q+t \pi / \alpha(y)<l-1+\varepsilon, a \in C^{\infty}(M)$, $\widehat{c}=\mathcal{X}_{c}, c \in \mathrm{H}^{l+k-1-\mu-\nu-k_{n}+q+|\gamma|+\varepsilon-t \pi / \alpha}(M)(q, t, \mu, \nu, k, l$ are non-negative integers). Then there exists a function

$$
\begin{aligned}
v=a(y) \widehat{c}(x) \partial_{y}^{\gamma}\left\{\frac{1}{4(\nu+1)} \partial_{z}^{q-1}\left[z^{\mu+t \pi / \alpha(y)} \bar{z}^{\nu} S_{\kappa}(z, \alpha(y))\right]\right. \\
+\sum_{j=0}^{n+1} b_{j}(y) z^{k_{n+1}-k_{j}+t \pi / \alpha(y)} S_{\kappa_{j}}(z, \alpha(y)) \\
\left.+\sum_{j=0}^{n+1} c_{j}(y) \bar{z}^{k_{n+1}-k_{j}+t \pi / \alpha(y)} S_{\kappa j}(z, \alpha(y))\right\}
\end{aligned}
$$


$\left(\kappa_{j}=\left(k_{0}, k_{1}, \ldots, k_{j}\right), k_{n+1}=k_{n}+\mu+\nu+2-q, b_{n+1} \equiv c_{n+1} \equiv 0\right.$ if $k_{n+1} \alpha^{*} / \pi$ is not integer) such that

$$
L v-f=T_{1}+T_{2}
$$

where

$$
T_{2} \in V_{-\varepsilon^{\prime}}^{l+k}(\mathcal{D}) \quad\left(\varepsilon^{\prime}=\varepsilon-k_{n} \sup _{y: \alpha(y)>\alpha^{*}}\left|\frac{\alpha^{*}}{\alpha(y)}-1\right|\right)
$$

and $T_{1}$ is a finite sum of expressions of the form

$$
b(x)\left(\partial_{y}^{\beta} \widehat{c}\right) \partial_{y}^{\gamma+\gamma^{\prime}} \partial_{x}^{p}\left\{z^{\mu^{\prime}+t \pi / \alpha(y)} \bar{z}^{\prime} S_{\kappa_{j}}(z, \alpha(y))\right\}
$$

and conjugate terms. Here

$$
\begin{aligned}
& p \leq \mu^{\prime}+\nu^{\prime}+1, \quad l-1+\varepsilon<\mu^{\prime}+\nu^{\prime}+k_{j}-p+t \pi / \alpha(y), \quad|\beta|+\left|\gamma^{\prime}\right| \leq 2 \\
& |\beta|+\left|\gamma^{\prime}\right| \leq\left(\mu^{\prime}+\nu^{\prime}+k_{j}-p\right)-\left(\mu+\nu+k_{n}-q\right), \quad b \in C^{\infty}(\mathcal{D}) .
\end{aligned}
$$

In particular, we have $D_{y}^{\beta}(L v-f) \in V_{-e}^{l}(\mathcal{D})$ if $|\beta| \leq k$. An analoguous representation holds for $q=0$ (cf Lemma \&).

The assertion follows immediately from Lemma 4 and the properties of the operator $\mathcal{K}$. In the proof one can use the fact that $L-4 \partial_{z} \partial_{\bar{z}}$ contains only terms of the form

$$
a\left(x_{1}, x_{2}, y\right) \partial_{x_{1}}^{i} \partial_{x_{2}}^{j} \partial_{y}^{\beta} \quad \text { where } i+j \leq 1, i+j+|\beta| \leq 2
$$

and

$$
a\left(x_{1}, x_{2}, y\right) \partial_{x_{1}}^{i} \partial_{x_{2}}^{j} \quad \text { where } i+j=2, a(0,0, y) \equiv 0
$$

b) The cases $\alpha^{*}=\pi, \alpha^{*}=2 \pi$. Assume that the angle $\alpha$ lies in a sufficiently small neighbourhood $U\left(\alpha^{*}\right)$ of the critical value $\alpha^{*}=\pi$ or $\alpha^{*}=2 \pi$. In these cases we can use a more simple form of the singular functions $S_{\kappa}(z, \alpha)$ because $k \alpha^{*} / \pi$ is integer for every integer $k$. Instead of $S_{k}(z, \alpha)$ for the tuple $\kappa=(0,1, \ldots, n)$ we will write $S_{n}(z, \alpha)$, i.e.

$$
S_{n}(z, \alpha) \equiv\left(\alpha-\alpha^{*}\right)^{-n} z^{n} \sum_{j=0}^{n}(-1)_{j}^{j} z^{j\left(\alpha^{*}-\alpha\right) / \alpha}=\left(\frac{z^{\alpha^{*} / \alpha}-z}{\alpha-\alpha^{*}}\right)^{n} .
$$

Again we consider at first the auxilliary problem

$$
\begin{aligned}
\partial_{z} \partial_{\bar{z}} v & =f_{0}=\partial_{z}^{g}\left\{z^{\mu+t \pi / \alpha} \bar{z}^{\nu} S_{n}(z, \alpha)\right\} & & \text { for } 0<\arg z<\alpha+\psi(r, \alpha) \\
v & =0 & & \text { for } \phi=\arg z=0 \text { and } \phi=\alpha+\psi(r, \alpha)
\end{aligned}
$$

where $\psi$ is a $C^{\infty}$-function on $\overline{\mathbb{R}}_{+} \times U\left(\alpha^{*}\right)$ such that $\psi(0, \alpha)=0$. We will construct a function $v$ (see Lemma 9) which satisfies the equations

$$
\begin{aligned}
\partial_{z} \partial_{\bar{z}} v & =f_{0} & & \text { for } 0<\arg z<\alpha+\psi(r, \alpha) \\
v & =0 & & \text { for } \arg z=0 \\
v & =0\left(r^{t \pi / \alpha^{-}+n+\mu+\nu+2-q+k-\varepsilon}\right) & & \text { for } \arg z=\alpha+\psi(r, \alpha)
\end{aligned}
$$

with an arbitrary given positive integer $k$. For this we need the following lemmas. 
Lemma 6. Let $v=z^{\mu+t \pi / \alpha} S_{n}(z, \alpha) \cdot(t, \mu$ integer $)$. Then the restriction of $v$ on the line $\phi=\alpha+\psi(r, \alpha)$ has the form

$$
\left.v\right|_{\phi=\alpha+\psi(r, \alpha)}=r^{\mu+t \pi / \alpha} \sum_{j=0}^{n} a_{j}(r, \alpha) r^{n-j} S_{j}(r, \alpha)
$$

where $a_{j} \in C^{\infty}\left(\dot{\bar{R}}_{+} \times U\left(\alpha^{*}\right)\right)$ for $j=0,1, \ldots, n$ and $a_{n}(r, \alpha)=(-1)^{t} e^{i n \alpha^{*}+i \mu \alpha}+$ $\widehat{a_{n}}(r, \alpha) \psi(r, \alpha), a_{n} \in C^{\infty}\left(\overline{R_{R}}+\times U\left(\alpha^{*}\right)\right)$.

Lemma 7. The restriction of the function

$$
v=\operatorname{Im}\left\{z^{t \pi / \alpha} \frac{z^{\mu \alpha^{*} / \alpha}-z^{\mu}}{\alpha-\alpha^{*}} S_{n}(z, \alpha)\right\}=\sum_{\nu=0}^{\mu-1} \operatorname{Im}\left\{z^{\mu-1-\nu+\left(t \pi+\nu \alpha^{*}\right) / \alpha} S_{n+1}(z, \alpha)\right\}
$$

on the line $\phi=\alpha+\psi(r, \alpha)$ has the form

$$
\begin{aligned}
& \left.v\right|_{\phi=\alpha+\psi(r, \alpha)}=(-1)^{t+1+n \alpha^{*} / \pi}\left\{\frac{\sin \mu \alpha}{\alpha-\alpha^{*}} r^{\mu+t \pi / \alpha}\right. \\
& \left.+n(-1)^{(\mu-1) \alpha^{*} / \pi} \frac{\sin \mu \alpha}{\alpha-\alpha^{*}} \sum_{\nu=0}^{\mu-1} r^{i \mu-\nu+\left(t \pi+\nu \alpha^{*}\right) / \alpha}\right\} S_{n}(r, \alpha) \\
& +\sum_{j=0}^{n-1} \sum_{\nu=0}^{\mu-1} a_{j \nu}(\alpha) r^{\mu-\nu+n-j+\left(t \pi+\nu \alpha^{*}\right) / \alpha} S_{j}(r, \alpha) \\
& +\sum_{j=0}^{n+1} \sum_{\nu=0}^{\mu-i} b_{j \nu}(r, \alpha) r^{\mu-\nu+n-j+1+\left(t \pi+\nu \alpha^{*}\right) / \alpha} S_{j}(r, \alpha)
\end{aligned}
$$

with coefficients $a_{j \nu} \in C^{\infty}(U(\alpha *))$ and $b_{j \nu} \in C^{\infty}\left(\overline{\mathbb{R}}_{+} \times U\left(\alpha^{*}\right)\right)$.

Proof. We have

$$
\begin{aligned}
\left.v\right|_{\phi=\alpha+\psi(r, \alpha)}= & (-1)^{t} \operatorname{Im}\left\{e^{i t \pi \psi / \alpha} r^{t \pi / \alpha}\left[e^{i \mu \alpha^{*}(1+\psi / \alpha)} \frac{r^{\mu \alpha^{*} / \alpha}-r^{\mu}}{\alpha-\alpha^{*}}+c_{\mu}(\alpha) r^{\mu}\right]\right. \\
& \left.\times\left[e^{i \alpha^{*}(1+\psi / \alpha)} S_{1}(r, \alpha)+c_{1}(\alpha) r\right]^{n}\right\}
\end{aligned}
$$

where

$$
c_{\mu}(\alpha)=\left(\alpha-\alpha^{*}\right)^{-1}\left(e^{i \mu \alpha^{*}(1+\psi / \alpha)}-e^{i \mu \alpha(1+\psi / \alpha)}\right) .
$$

Using the fact that $\psi(r, \alpha) / r \in C^{\infty}\left(\overline{\mathbb{R}}_{+} \times U\left(\alpha^{*}\right)\right)$. we obtain the assertion of the lemma

Corollary 1. Let $g=c(\alpha) r^{\mu+t \pi / \alpha} S_{n}(z, \alpha)$ where $t, \mu$ are non-negative integers, $\mu \geq 1$ and $c$ is a $C^{\infty}$-function on $U\left(\alpha^{*}\right)$ satisfying the condition $c\left(\alpha^{*}\right) \neq 0$. Then there exists a function

$$
v=\sum_{\nu=0}^{\mu-1} c_{\nu}(\alpha) \operatorname{Im}\left\{z^{\mu-\nu-1+\left(t \pi+\nu \alpha^{*}\right) / \alpha} S_{n+1}(z, \alpha)\right\} \quad\left(c_{\nu} \in C^{\infty}\left(U\left(\alpha^{*}\right)\right)\right.
$$


such that the restriction of $v$ on the line $\phi=\alpha+\psi(r, \alpha)$ satisfies the equation

$$
\begin{aligned}
\left.v\right|_{\phi=\alpha+\psi}-g= & \sum_{j=0}^{n-1} \sum_{\mu=0}^{\mu-1} a_{j \nu}(\alpha) r^{\mu-\nu+n-j+\left(t \pi+\nu \alpha^{*}\right) / \alpha} S_{j}(r, \alpha) \\
& +\sum_{j=0}^{n+1} \sum_{\nu=0}^{\mu-1} b_{j \nu}(r, \alpha) r^{\mu-\nu+n+1-j+\left(t \pi+\nu \alpha^{*}\right) / \alpha} S_{j}(r, \alpha)
\end{aligned}
$$

with coefficients $a_{j \nu} \in C^{\infty}\left(U\left(\alpha^{*}\right)\right)$ and $b_{j \nu} \in C^{\infty}\left(\bar{R}_{+} \times U\left(\alpha^{*}\right)\right)$.

Proof. The corollary can be easily shown by induction on $\mu$. For $\mu=1$ the corresponding result follows immediately from Lemma 7. Assume that the assertion is true for $\mu-1(\mu \geq 2)$. Let

$$
d(\alpha)=c_{0}(\alpha)(-1)^{t+1+n \alpha^{*} / \pi}\left(\alpha-\alpha^{*}\right) /\left(\sin (\mu \alpha)+n(-1)^{(\mu-1) \alpha^{*} / \pi} \sin \alpha\right)
$$

(i.e. $\left.d\left(\alpha^{*}\right)=(-1)^{t+1+(n+\mu) \alpha^{\bullet} / \pi} c_{0}(\alpha) /(n+\mu)\right)$ and

$$
v_{1}=d(\alpha) \sum_{\nu=0}^{\mu-1} \operatorname{Im}\left\{z^{\mu-\nu-1+\left(1 \pi+\nu \alpha^{*}\right) / \alpha} S_{n+1}(z, \alpha)\right\}
$$

Then Lemma 7 implies

$$
\begin{aligned}
\left.v_{1}\right|_{\phi=\alpha+\psi}-g= & d(\alpha) n(-1)^{t+1+(n+\mu-1) \alpha^{*} / \pi} \\
& \times \frac{\sin \alpha}{\alpha-\alpha^{*}} \sum_{n=1}^{\mu-1} r^{\mu-\nu+\left(t \pi+\nu \alpha^{*}\right) / \alpha} S_{n}(r, \alpha) \\
& +\sum_{j=0}^{n-1} \sum_{\nu=0}^{\mu-1} a_{j \nu}(\alpha) r^{\mu-\nu+n-j+\left(t \pi+\nu \alpha^{*}\right) / \alpha} S_{j}(r, \alpha) \\
& +\sum_{j=0}^{n+1} \sum_{\nu=0}^{\mu-1} b_{j \nu}(r, \alpha) r^{\mu-\nu+n+1-j+\left(\imath \pi+\nu \alpha^{*}\right) / \alpha} S_{j}(r, \alpha) .
\end{aligned}
$$

By our assumption there exists a function $v_{2}$ of the form (2.5) such that

$$
\left.v_{2}\right|_{\phi=\alpha+\psi}-d(\alpha) n(-1)^{t+1+(n+\mu-1) \alpha^{*} / \pi} \frac{\sin \alpha}{\alpha-\alpha^{*}} \sum_{\nu=1}^{\mu-1} r^{\left(t \pi+\nu \alpha^{*}\right) / \alpha+\mu-\nu} S_{n}(r, \alpha)
$$

is an expression of the form (2.6). Then the difference $v=v_{1}-v_{2}$ satisfies the condition of Corollary 1

Lemma 8. Let

$$
g=c(\alpha) r^{\mu+t \pi / \alpha} S_{n}(r, \alpha)
$$


with non-negative integers $t, \mu, \nu, \mu \geq 1$, and $c \in C^{\infty}\left(U\left(\alpha^{*}\right)\right)$. Then there exists a function

$$
v=\sum_{j=0}^{n+1} \sum_{\nu=0}^{\mu+n-j} c_{j \nu}(r, \alpha) \operatorname{Im}\left\{z^{\mu-\nu+n-j+\left(t+i+\nu \alpha^{*}\right) / \alpha} S_{j}(z, \alpha)\right\}
$$

$\left(c_{j \nu} \in C^{\infty}\left(U\left(\alpha^{*}\right)\right)\right)$ such that

$$
\left.v\right|_{\phi=\alpha+\psi}-g=\sum_{j=0}^{n+1} \sum_{\nu=0}^{\mu+n-j} a_{j \nu}(r, \alpha) r^{\mu-\nu+n+1-j+\left(t \pi+\nu \alpha^{*}\right) / \alpha} S_{j}(r, \alpha)
$$

where the coefficients $a_{j \nu}$ are functions from $C^{\infty}\left(\overline{\mathbb{R}}_{+} \times U\left(\alpha^{*}\right)\right)$.

Proof. We prove the lemma by induction in $n$. Let at first $n=0$, i.e. $g=$ $c(\alpha) r^{\mu+t \pi / \alpha}$. If $c\left(\alpha^{*}\right)=0$, then the assertion is true for $v=(-1)^{t} \frac{c(\alpha)}{\sin \mu \alpha} \operatorname{Im} z^{\mu+t \pi / \alpha}$, and if $c\left(\alpha^{*}\right) \neq 0$, we set

$$
\begin{aligned}
v & =(-1)^{t+1} \frac{\alpha-\alpha^{*}}{\sin \mu \alpha} c(\alpha) \operatorname{Im}\left\{z^{t \pi / \alpha} \frac{z^{\mu \alpha^{*} / \alpha}-z^{\mu}}{\alpha-\alpha^{*}}\right\} \\
& =(-1)^{t+1} \frac{\alpha-\alpha^{*}}{\sin \mu \alpha} c(\alpha) \sum_{\nu=0}^{\mu-1} \operatorname{Im}\left\{z^{\mu-\nu-1+\left(t \pi+\nu \alpha^{*}\right) / \alpha} S_{1}(z, \alpha)\right\} .
\end{aligned}
$$

We now assume that the assertion is proved for $n-1$ and show its validity for $n$. Again we have to consider two different cases $c\left(\alpha^{*}\right)=0$ and $c\left(\alpha^{*}\right) \neq 0$. Consider at first the case $c\left(\alpha^{*}\right)=0$. If we set

$$
v_{1}=(-1)^{t+n \alpha \cdot / \pi} \frac{c(\alpha)}{\sin \mu \alpha} \operatorname{Im}\left\{z^{\mu+t \pi / \alpha} S_{n}(z, \alpha)\right\}
$$

then we get

$$
\left.v\right|_{\phi=\alpha+\psi(r, \alpha)}-g=g_{1}+g_{2}
$$

where

$$
g_{1}=\sum_{j=0}^{n-1} d_{j}(\alpha) r^{\mu+n-j+t \pi / \alpha} S_{j}(r, \alpha) \quad \text { and } \quad g_{2}=d(r, \alpha) r^{\mu+1+t \pi / \alpha} S_{n}(r, \alpha)
$$

with some smooth coefficients $d_{j}$ and $d$ (see Lemma 6). By our assumption there exists a function

$$
v_{2}=\sum_{j=0}^{n} \sum_{\nu=0}^{\mu+n-j} c_{j \nu}(\alpha) \operatorname{Im}\left\{z^{\mu-\nu+n-j+\left(t \pi+\nu \alpha^{*}\right) / \alpha} S_{j}(z, \alpha)\right\}
$$

such that

$$
\left.v_{2}\right|_{\phi=\alpha+\psi}-g_{1}=\sum_{j=0}^{n} \sum_{\nu=0}^{\mu+n-j} a_{j \nu}(r, \alpha) r^{\mu-\nu+n+1-j+\left(t \pi+\nu \alpha^{\bullet}\right) / \alpha} S_{j}(r, \alpha)
$$

with coefficients $a_{j \nu} \in C^{\infty}\left(\overline{I R}_{+} \times U\left(\alpha^{*}\right)\right)$. Then $v=v_{1}-v_{2}$ satisfies the condition of the lemma. In the case $c\left(\alpha^{*}\right) \neq 0$ the assertion can be analogously proved by means of Corollary 1 
Corollary 2. Let

$$
g=c(\alpha) r^{\mu+t \pi / \alpha} S_{n}(r, \alpha) \quad\left(t, \mu, \nu \in N_{0}, \mu \geq 1, c \in C^{\infty}\left(U\left(\alpha^{*}\right)\right)\right)
$$

Then for every integer $k \geq 1$ there exists a function

$$
v=\sum_{i=1}^{k} \sum_{j=0}^{n+i \mu+n+i-j-1} \sum_{\nu=0} c_{i j \nu}(\alpha) \operatorname{Im}\left\{z^{\mu-\nu+n+i-j-1+\left(t \pi+\nu \alpha^{\bullet}\right) / \alpha} S_{j}(z, \alpha)\right\}
$$

with coefficients $c_{i j \nu} \in C^{\infty}\left(U\left(\alpha^{*}\right)\right)$ such that

$$
\left.v\right|_{\phi=\alpha+\psi(r, \alpha)}-g=\sum_{j=0}^{n+k} \sum_{\nu=0}^{\mu+n+k-j-1} a_{j \nu}(r, \alpha) r^{\mu-\nu+n-j+k+\left(t \pi+\nu \alpha^{*}\right) / \alpha} S_{j}(r, \alpha)
$$

where the coefficients $a_{j \nu}$ are smooth functions on $\overline{\mathbb{R}}_{+} \times U\left(\alpha^{*}\right)$.

We now can show an analogon to Lemma 4:

Lemma 9. Let $f_{0}=\partial_{z}^{q}\left\{z^{\mu+\imath \pi / \alpha} \bar{z}^{\nu} S_{n}(z, \alpha)\right\}$ where $t, \mu, \nu, n \in N_{0}, \mu+\nu \geq q-1$. Then for every given integer $k \geq 1$ there exists a function

$$
\begin{aligned}
v= & \sum_{j=0}^{n} A_{j}(\alpha) z^{\mu+1-q+n-j} \bar{z}^{\nu+1} S_{j}(z, \alpha) \\
& +\sum_{j=0}^{n} B_{j}(\alpha) \operatorname{Re}\left\{z^{\mu-j+t \pi / \alpha^{\prime}} S_{j}(z, \alpha)\right\} \\
& +\sum_{i=0}^{k-1} \sum_{j=0}^{n+1} \sum_{s=0}^{m+i-j} C_{i j,}(\alpha) \operatorname{Im}\left\{z^{m+i-j-s+\left(1 \pi+s \alpha^{*}\right) / \alpha} S_{j}(z, \alpha)\right\}
\end{aligned}
$$

where $m=n+\mu+\nu+2-q$ and the coefficients $A_{j}, B_{j}, C_{i j}$, belong to the space $C^{\infty}\left(U\left(\alpha^{*}\right)\right)$ such that

$$
\begin{aligned}
\partial_{z} \partial_{\bar{z}} v & =f \quad \text { for } 0<\phi=\arg z<\alpha+\psi(r, \alpha) \\
v & =0 .
\end{aligned}
$$

and

$$
v=\sum_{j=0}^{n+k-1} \sum_{j=0}^{m+k-1-j} a_{j s}(r, \alpha) r^{m+k-j-s+\left(t \pi+s \alpha^{*}\right) / \alpha} S_{j}(r, \alpha) \text { for } \phi=\alpha+\psi(r, \alpha)
$$

Here the coefficients $a_{j}$, are functions from $C^{\infty}\left(\overline{\mathbb{R}}_{+} \times U\left(\alpha^{*}\right)\right)$. In the case $q \geq 1$ the first term in (2.7) can be replaced by $\frac{1}{\nu+1} \bar{z}^{\nu+1} \partial_{z^{q-1}}^{q}\left\{z^{\mu+t \pi / \alpha} S_{n}(z, \alpha)\right\}$. 
Proof. By Lemma 4/Remark 1 there exists a function

$$
\begin{aligned}
v_{1}= & \sum_{j=0}^{n} A_{j}(\alpha) z^{\mu+1-q+n-j+t \pi / \alpha} \bar{z}^{\nu+1} S_{j}(z, \alpha) \\
& +\sum_{j=0}^{n} B_{j}(\alpha) \operatorname{Re}\left\{z^{m-j+t \pi / \alpha} S_{j}(z, \alpha)\right\} \\
& +\sum_{j=0}^{n} C_{j}(\alpha) \operatorname{Im}\left\{z^{m-j+t \pi / \alpha} S_{j}(z, \alpha)\right\}
\end{aligned}
$$

satisfying the equations $\partial_{z} \partial_{\bar{z}} v_{1}=f$ and $\left.v_{1}\right|_{\phi=0}=\left.v_{1}\right|_{\phi=\alpha}=0$. Hence, the restriction of $v_{1}$ on the line $\phi=\alpha+\psi(r, \alpha)$ has the form

$$
\left.v_{1}\right|_{\phi=\alpha+\psi(r, \alpha)}=\sum_{j=0}^{n} a_{j}(r, \alpha) r^{m+1-j+t \pi / \alpha} S_{j}(r, \alpha)
$$

with coefficients $a_{j} \in C^{\infty}\left(\overline{\mathbb{R}}_{+} \times U\left(\alpha^{*}\right)\right)$. Corollary 2 implies the existence of a function

$$
v_{2}=\sum_{i=1}^{k-1} \sum_{j=0}^{n+i} \sum_{s=0}^{m+i-j} c_{i j}(\alpha) \operatorname{Im}\left\{z^{m-s+i-j+\left(t \pi+s \alpha^{*}\right) / \alpha} S_{j}(z, \alpha)\right\}
$$

such that

$$
v_{1}+\left.v_{2}\right|_{\phi=\alpha+\psi(r, \alpha)}=\sum_{j=0}^{n+k-1} \sum_{s=0}^{m+k-1-j} a_{j s}(r, \alpha) r^{m+k-s-j+\left(t \pi+\varepsilon \alpha^{*}\right) / \alpha} S_{j}(r, \alpha) .
$$

Hence, $v=v_{1}+v_{2}$ satisfies the conditions of the lemma

Now we consider again problem (1.2) in the dihedron

$$
\mathcal{D}=\left\{x=\left(x_{1}, x_{2}, y\right) \in \mathbb{R}^{N}: y \in \mathbb{R}^{N-2}, 0<\phi=\arg \left(x_{1}+i x_{2}\right)<\omega(r, y)\right\}
$$

where $\omega(r, y)=\alpha(y)+\psi(r, y)$ and $\alpha(y)=\omega(0, y)$ lies in a sufficiently small neighbourhood $U\left(\alpha^{*}\right)$ of the critical angle $\alpha^{*}=\pi$ or $\alpha^{*}=2 \pi$. We denote the sides $\phi=0$ and $\phi=\omega(r, y)$ of $\mathcal{D}$ by $\Gamma_{-}$and $\Gamma_{+}$, respectively. By means of the last lemma and the properties of the extension operator $\mathcal{K}$ one obtains the following proposition analogously to Proposition 2.

Proposition 3. Let $l$ and $k$ be arbitrary positive integers and let $f$ be a function of the form

$$
f=a(y) \widetilde{c}(x) \partial_{y}^{\gamma} \partial_{z}^{q}\left\{z^{\mu+t \pi / \alpha(y)} \bar{z}^{\nu} S_{n}(z, \alpha(y))\right\}
$$

where

$$
\begin{aligned}
& n \leq l-1, q \leq \mu+\nu+1, l-2+\varepsilon<n+\mu+\nu-q+t \pi / \alpha(\cdot)<l-1+\varepsilon \\
& a \in C^{\infty}(M), \widehat{c}=\dot{K} c, c \in H^{l+k-1-n-\mu-\nu+q+|\gamma|+\varepsilon-\imath \pi / \alpha(\cdot)}(M)
\end{aligned}
$$


$\left(q, t, n, \mu, \nu\right.$ are non-negative integers, $\varepsilon>0$, sufficiently small). Let furthermore $\varepsilon^{\prime}$ be an arbitrary positive real number less than $\varepsilon$. Assume that $\alpha(y)$. lies in a sufficiently small neighbourhood $U\left(\alpha^{*}\right)$ of $\alpha^{*}$ (which depends on $l, k$ and $\varepsilon-\varepsilon^{\prime}$ ) for all $y \in M$. Then there exists a function

$$
\begin{aligned}
v= & a(y) \widehat{c}(x) \partial_{y}^{\gamma}\left(\sum_{j=0}^{n} a_{j}(y) z^{\mu+1-q+n-j+t \pi / \alpha(y)} \bar{z}^{\nu+1} S_{j}(z, \alpha)\right. \\
& +\sum_{j=0}^{n} b_{j}(y) \operatorname{Re}\left[z^{m-j+t \pi / \alpha(y)} S_{j}(z, \alpha)\right] \\
& \left.+\sum_{i=0}^{k-1} \sum_{j=0}^{n+i} \sum_{j=0}^{m+i-j} c_{i j o}(y) \operatorname{Im}\left[z^{m+i-j-s+\left(t \pi+s \alpha^{*}\right) / \alpha} S_{j}(z, \alpha)\right]\right)
\end{aligned}
$$

where $m=n+\mu+\nu+2-q$ and the coefficients $a_{j}, b_{j}, c_{i j}$ are smooth functions on $M$ such that

$$
L v-f=T_{1}+T_{2} \text { in } \mathcal{D},\left.\quad v\right|_{\Gamma_{-}}=0,\left.\quad v\right|_{\Gamma_{+}} \in V_{-\varepsilon^{\prime}}^{l+k-1 / 2}\left(\Gamma_{+}\right) .
$$

Here $T_{2} \in V_{-e^{\prime}}^{l+k}(\mathcal{D})$ and $T_{1}$ is a finite sum of expressions

$$
b(x)\left(\partial_{y}^{\beta} \widehat{c}\right) \partial_{y}^{\gamma+\gamma^{\prime}} \partial_{z}^{p}\left\{z^{\left.\left.\mu^{\prime}-\right\lrcorner+(t \pi+\lrcorner \alpha^{\bullet}\right) / \alpha} \bar{z}^{\mu} S_{j}(z, \alpha)\right\}
$$

and conjugate terms where

$$
\begin{aligned}
& p \leq \mu^{\prime}+\nu^{\prime}-s+1, \quad \mu^{\prime}+\nu^{\prime}+j-p+t \pi / \alpha>l-1+\varepsilon, \quad j \leq n+k-1 \\
& s \leq \mu^{\prime}+\nu^{\prime}+1, \quad n+\mu+\nu+1-q \leq \mu^{\prime}+\nu^{\prime}+j \leq n+\mu+\nu+k-q^{\prime}
\end{aligned}
$$

and $|\beta|+\left|\gamma^{\prime}\right| \leq 2$.

\section{Asymptotics of the solution in a neighbourhood 'of an edge}

Let $G$ be a bounded domain in $\mathbb{R}^{N}$ which coincides with the dihedron (1:1) in a neighbourhood $\mathcal{V}$ of the point $y_{0} \in M$. We consider the Dirichlet problem

$$
\begin{aligned}
L u & =\sum_{i+j+|\beta| \leq 2} a_{i j \beta}(x) \partial_{x_{1}}^{i} \partial_{x_{2}}^{j} \partial_{y}^{\beta} u=f \text { in } \\
u & =0
\end{aligned}
$$

for the elliptic differential operator $L$ with real-valued coefficients $a_{i j \beta} \in C^{\infty}(\bar{G})$. Again without loss of generality we may assume that the condition (1.3) is satisfied for every point $(0,0, y) \in \mathcal{V} \cap M$. Suppose that the angle $\alpha(y)$ in the point $y_{0}$ is equal to a critical value $\alpha^{*}$, i.e. there exists a number $k \in\{1,2, \ldots, l+1\}$ such that $k \alpha^{*} / \pi$ is integer. In the case $\alpha^{*} \neq \pi, \alpha^{*} \neq 2 \pi$ we assume that the function $\omega$ in the definition of the 
dihedron $\mathcal{D}$ does not depend on the variable $r$. Moreover, let $\varepsilon^{\prime}<\varepsilon$ be sufficiently small positive numbers (such that the assertion of Proposition 1 is valid) satisfying the condition

$$
t \pi / \alpha^{*} \notin\left[\mu+\varepsilon^{\prime}, \mu+\varepsilon\right]
$$

for every non-negative integer $\mu$ and $t, \mu \leq l+1$. We further denote by $U\left(\alpha^{*}\right)$ a sufficiently small neighbourhood of $\alpha^{*}$ which depends on $l, \varepsilon$ and $\varepsilon^{\prime}$. In particular, we assume that $\alpha^{*}$ is the only critical angle in $U\left(\alpha^{*}\right)$ and that $t \pi / \alpha \notin\left[\mu+\varepsilon^{\prime}, \mu+\varepsilon\right]$ for every $\alpha \in U\left(\alpha^{*}\right)$ and every non-negative integer $\mu, t, \mu \leq l+1$. Then we define the neighbourhood $\mathcal{V}_{0} \subseteq \mathcal{V}$ as

$$
\mathcal{V}_{0}=\left\{x=\left(x_{1}, x_{2}, y\right) \in \mathcal{V}: \alpha(y) \in U\left(\alpha^{*}\right)\right\}
$$

The following lemma has been shown in effect, e.g., in [9], [10] for $\alpha^{*} \neq \pi, \alpha^{*} \neq 2 \pi$. By means of Theorem 1 this lemma can be proved in a similar way for arbitrary $\alpha^{*}$.

Lemma 10. Let $u \in V_{-\varepsilon}^{l+1}(G)$ be a solution of problem (9.1) with support in $V_{0} \cap \dot{\bar{G}}$. Suppose that the right-hand side of (9.1) satisfies the condition $D_{y}^{\beta} f \in V_{-e+k-|\beta|}^{l+k-|\beta|}(\mathcal{D})$ for $|\beta| \leq k$. Then

$$
D_{y}^{\beta} u \in V_{-\varepsilon+k+1-|\beta|}^{l+k+2-|\beta|}(\mathcal{D}) \subset V_{-\varepsilon}^{l+1}(\mathcal{D}) \quad \text { for }|\beta| \leq k+1
$$

and $u$ admits the decomposition

$$
u=\sum_{t \in I_{t}} \widehat{c_{t}}(r, y) \operatorname{Im} z^{t \pi / \alpha(y)}+u_{0}
$$

where $I_{l}=\left\{t: t\right.$ integer, $\left.l+\varepsilon<t \pi / \alpha^{*}<l+1+\varepsilon\right\}, D_{y}^{\beta} u_{0} \in V_{-e^{\prime}+k+1-|\beta|}^{l+k+3-|\beta|}(\mathcal{D})$ for $|\beta| \leq k, \widehat{c_{t}}=\mathcal{K} c_{t} ; c_{t} \in W^{l+k+1+\varepsilon^{\prime}-t \pi / \alpha(\cdot)}(M)$.

Proof. In the case $l=0$ the assertion $D_{y}^{\beta} u \in V_{-e+k+1-|\beta|}^{k+2-|\beta|}(\mathcal{D})$ follows from Theorem 1 and the decomposition (3.2) holds analogously to Theorem 4.1, [10: Remark 4.1] and [9: Lemma 3.6]. We now assume that Lemma 10 is proved for $l-1$ and show the validity of this lemma for a given $l \geq 1$. For simplicity we restrict ourselves to the case $N=3$. Lemma 1 implies $u \in V_{-\varepsilon+k+1}^{l+k+2}(\mathcal{D})$. Let $D_{1}=\partial_{y}+\frac{\partial_{y} \omega(r, y)}{\omega(r, y)} \varphi \partial_{\varphi}$ be the operator (1.5). Then the function $v=D_{1} u$ satisfies the equations

$$
L v=F \quad \text { in } \mathcal{D}, \quad v=0{ }^{\circ} \text { on } \partial \mathcal{D}
$$

where $F=L\left(D_{1} u\right)=D_{1} L u+L_{1} u=D_{1} f+L_{1} u$ and $L_{1}$ is an operator of the form (1.6). Obviously, $v \in V_{-e+k+1}^{l+k+1}(\mathcal{D}) \subset V_{-c}^{l}(\mathcal{D}), F \in V_{-e+k}^{l+k-1}(\mathcal{D}) \subset V_{-\varepsilon}^{l-1}(\mathcal{D})$ and our assumption implies $\partial_{y} v \in V_{-e}^{l}(\mathcal{D})$. Using Lemma 1 one gets $\partial_{y} v \in V_{-e+k}^{l+k}(\mathcal{D})$. By induction in $k$ we can further show that $\partial_{y}^{j} F \in V_{-\varepsilon+k-j}^{1-1+k-j}(D)$ for $j=1, \ldots, k$ and therefore (by our assumption) $\partial_{y}^{j} v \in V_{-\varepsilon+k+1-j}^{1+k+1-j}(\mathcal{D})$ for $j=1, \ldots, k+1$. Furthermore, we obtain

$$
\dot{v}=\sum_{t \in I_{1-1}} \hat{\hat{c}_{t}} \operatorname{Im} z^{t \pi / \alpha}+v_{0}
$$


where

$\partial_{y}^{j} v_{0} \in V_{-e^{\prime}+k-j}^{t+k+1-j}(\mathcal{D})$ for $j=0,1, \ldots, k, \widehat{c_{t}}=\mathcal{K} c_{t}, c_{t} \in W^{l+k+e^{\prime}-t \pi / \alpha}(M)$.

Integrating (3.3) we get

$$
\int_{y_{1}}^{y}\left(v\left(x_{1}, x_{2}, s\right)-v_{0}\left(x_{1}, x_{2}, s\right)\right) d s=\sum_{t \in I_{1-1}} \int_{y_{1}}^{y} \widehat{c_{t}}(r, s) \operatorname{Im} z^{t \pi / a(s)} d s .
$$

Here $y_{1}$ is a point on $M \cap \mathcal{V}_{0}$ such that $u\left(x_{1}, x_{2}, y\right)=0$ for $y<y_{1}$. Since the function

$$
\begin{aligned}
& \int_{y_{1}}^{y}\left(v\left(x_{1}, x_{2}, s\right)-v_{0}\left(x_{1}, x_{2}, s\right)\right) d s \\
& \quad=u\left(x_{1}, x_{2}, y\right)+\int_{y_{1}}^{y}\left[\frac{\partial, \omega(r, s)}{\omega(r, s)} \phi \partial_{\phi} u\left(x_{1}, x_{2}, s\right)-v_{0}\left(x_{1}, x_{2}, s\right)\right] d s
\end{aligned}
$$

belongs to $V_{-e^{\prime}-l-1}^{0}(\mathcal{D})$ we get $c_{t}=0$ a. e. on $M$, i: e. $v=v_{0}$ and $\partial_{y}^{j} v \in V_{-e^{\prime}+k-j}^{l+k+1-j}(\mathcal{D})$ for $j \stackrel{=}{=} 0,1, \ldots, k$. It can be shown by induction in $k$ that

$$
L\left(\partial_{y}^{j} v\right)=\partial_{y}^{j} L v+\sum_{\nu=0}^{j-1} L_{\nu}\left(\partial_{y}^{\nu} v\right) \in V_{-e+k-j}^{l+k-1-j}(\mathcal{D})
$$

(here $L_{\nu}$ are certain second order differential operators with smooth coefficients) and therefore

$$
\partial_{v}^{j} v \in V_{-\epsilon+k-j}^{l+k+1-\ddot{D}}(\mathcal{D}) \quad \text { for } j=0,1, \ldots, k \text {. }
$$

This implies

$$
\partial_{y}^{j} u \in V_{-e+k+1-j}^{l+k+2-j}(\mathcal{D}) \quad \text { for } j=0,1, \ldots, k+1 \text {. }
$$

Analogously to Theorem 4.1, [10: Remark 4.1] and [9: Lemma 3.6] one can show the validity of the decomposition (3.2)

Proposition 4. Let $\alpha^{*} \neq \pi, \alpha^{*} \neq 2 \pi$ and let $u \in \dot{W}^{1}(G)$ be a solution of problem (9.1) with $f \in W_{-e}^{l+k}(\mathcal{D})$. Then $u$ has the following representation in $\mathcal{V} \cap G$ :

$$
u=\Sigma_{l}+u_{l}
$$

where

$$
u_{l}=0 \text { on } \partial \mathcal{D} \cap \mathcal{V}_{0} \quad \text { and } \quad D_{y}^{\beta} u_{l} \in V_{-c^{\prime}+k-|\beta|}^{1+k+2-|\beta|}(\mathcal{D}) \text { for }|\beta| \leq k
$$

and $\Sigma_{l}$ is a finite sum of expressions of the form

$$
\widetilde{c}(x) \partial_{y}^{\gamma} \partial_{z}^{p}\left\{z^{\mu+t \pi / a} \bar{z}^{\nu} S_{\kappa}(z, \alpha)\right\}
$$


and conjugate terms. Here $t, p, \mu, \nu$ are non-negative integers, $p \leq \mu+\nu, p \leq l, \kappa=$ $\left(k_{0}, k_{1}, \ldots, k_{n}\right) \in \mathcal{K}_{l}\left(\alpha^{*}\right), \mu+\nu+k_{n}-p+t \pi / \alpha^{*}<l+1+\varepsilon$ and $\widehat{c}=\mathcal{K} c$ with

$$
c \in W^{l+k+1+|\gamma|-\mu-\nu-k_{n}+p+e^{\prime}-t \pi / \alpha(\cdot)}(M)
$$

Furthermore, $L \Sigma_{l}-P_{l}(f)$ is the sum of a function $f_{0} \in V_{-\varepsilon^{\prime}}^{l+k}(\mathcal{D})$ and a finite number of expressions of the form

$$
a(x) \widehat{c}(x) \partial_{y}^{\gamma} \partial_{z}^{p}\left\{z^{\mu+t \pi / \alpha} \bar{z}^{\nu} S_{\kappa}(z, \alpha)\right\}
$$

and conjugate terms $\left(\kappa=\left(k_{0}, k_{1}, \ldots, k_{n}\right) \in \mathcal{K}_{l}\left(\alpha^{*}\right), p \leq \mu+\nu+1, p \leq l+2, l-1+\varepsilon^{\prime}<\right.$ $\left.\mu+\nu+k_{n}-p+t \pi / \alpha^{*}<l+k-1+\varepsilon^{\prime}\right)$ with

$$
\widehat{c}=\mathcal{K} c, \quad c \in W^{l+k-1+|\gamma|-\mu-\nu-k_{n}+p+\varepsilon^{\prime}-t \pi / \alpha(\cdot)}(M) .
$$

Proof. We prove the proposition by induction in $l$. Let $\varepsilon_{1}, \ldots, \varepsilon_{l+1}$ be a suitable sequence of positive numbers such that $\varepsilon^{\prime}<\varepsilon_{l+1}<\varepsilon_{l}<\cdots<\varepsilon_{1}<\epsilon$. We consider at first the case $l=0$. If $\pi / \alpha^{*}>1+\varepsilon_{1}$, then $u \in V_{-e_{1}}^{2}\left(G \cap \mathcal{V}_{0}\right)$. Furthermore, Lemma 10 implies $D_{y}^{\beta} u \in V_{-e_{1}}^{2}\left(G \cap \mathcal{V}_{0}\right)$ for $|\beta| \leq k$. If $\pi / \alpha^{*}<1+\varepsilon_{1}$, then

$$
u=\widehat{c_{1}}(x) \operatorname{Im} z^{\pi / \alpha(y)}+u_{0}
$$

where

$$
\widehat{c_{1}}=\mathcal{K} c_{1}, \quad c_{1} \in W^{k+1+\varepsilon_{1}-\pi / \alpha}(M), \quad D_{y}^{\beta} u_{0} \in V_{-\varepsilon_{1}+k-|\beta|}^{2+k-|\beta|}(\mathcal{D}) \text { for }|\beta| \leq k
$$

and $u_{0}=0$ on $\partial \mathcal{D} \cap \mathcal{V}_{0}$. Moreover,

$$
L\left(\hat{c_{1}} \operatorname{Im} z^{\pi / \alpha}\right)=\left[L, \widehat{c_{1}}\right] I m z^{\pi / \alpha}+\widehat{c_{1}}\left(L-4 \partial_{z} \partial_{\bar{z}}\right) \operatorname{Im} z^{\pi / \alpha}=T_{1}+T_{2}
$$

$\left(\left[L, \widehat{c_{1}}\right]\right.$ denotes the commutator of $L$ and $\left.\widehat{c_{1}}\right)$ where $T_{2} \in V_{-e_{1}}^{k}(\mathcal{D})$ and $T_{1}$ is a finite sum of expressions of the form

$$
a(x)\left(\partial_{y}^{\gamma} \widehat{c_{1}}\right) \partial_{y}^{\gamma^{\prime}} \partial_{z}^{p}\left(z^{\mu+\pi / \alpha} \bar{z}^{\nu}\right)
$$

and conjugate terms where $a \in C^{\infty}\left(\bar{G} \cap \mathcal{V}_{0}\right), \mu, \nu, p \in \mathbb{N},|\gamma|+\left|\gamma^{\prime}\right|+p \leq 2, p \leq \mu+\nu+1 \leq$ 2. Hence the assertion is true for $l=0$.

We prove the assertion for arbitrary 1 . For this purpose we assume the assertion to be true for $l-1$. This assumption implies

$$
u=\Sigma_{l-1}+u_{l-1}
$$

where

a) $D_{y}^{\beta} u_{1-1} \in V_{-e_{1}}^{l+1}(\mathcal{D})$ for $|\beta| \leq k+1$.

b) $\Sigma_{l-1}$ is a finite sum of expressions of the form $\widetilde{c}(x) \partial_{y}^{\gamma} \partial_{z}^{p}\left\{z^{\mu+t \pi / \alpha} \bar{z}^{\nu} S_{\kappa}(z, \alpha)\right\}$ and conjugate terms $\left(\kappa=\left(k_{0}, \ldots, k_{n}\right) \in \mathcal{K}_{l-\mathrm{i}}\left(\alpha^{*}\right), p \leq \mu+\nu, p \leq l-1, \mu+\nu+k_{n}-p+t \pi / \alpha^{*}<\right.$ $\left.l+\varepsilon, \widehat{c}=\mathcal{K} c, c \in W^{l+k+1+|\gamma|-\mu-\nu-k_{n}+p+\varepsilon_{l}-t \pi / \alpha(\cdot)}(M)\right)$. 
c) $L \Sigma_{l-1}-P_{l-1}(f)=A_{1}+A_{2}$. Here $A_{2} \in V_{-e_{l}}^{l+k}\left(\mathcal{D} \cap \mathcal{V}_{0}\right)$ and $A_{1}$ is a finite sum of expressions

$$
a(x) \widetilde{c}(x) \partial_{y}^{\gamma} \partial_{z}^{p}\left\{z^{\mu+\imath \pi / \alpha} \bar{z}^{\nu} S_{\kappa}(z, \alpha)\right\}
$$

and conjugate terms $\left(\kappa=\left(k_{0}, \ldots, k_{n}\right) \in \mathcal{K}_{l-1}\left(\alpha^{*}\right), l-2+\varepsilon_{l}<\mu+\nu+k_{n}-p+t \pi / \alpha^{*}<\right.$ $\left.l+k-1+\varepsilon_{l}, \widehat{c}=\mathcal{K}_{c}, c \in W^{l+k-1+|\gamma|-\mu-\nu-k_{n}+p+e_{1}-t \pi / \alpha(\cdot)}(M)\right)$.

From the properties of the operator $\mathcal{K}$ (see Lemma 5 ) it follows that $A_{1}=A_{1}{ }^{\prime}+A_{2}{ }^{\prime \prime}$ where $D_{y}^{\beta} A_{1}{ }^{\prime \prime} \in V_{-e^{\prime}}^{\prime}(\mathcal{D})$ for $|\beta| \leq k$ and $A_{1}{ }^{\prime}$ is a finite sum of terms

$$
a(0,0, y) \widehat{c} \partial_{y}^{\gamma} \partial_{z}^{p}\left\{z^{\mu+i \pi / \alpha} \bar{z}^{\nu} S_{\kappa}(z, \alpha)\right\}
$$

and conjugate terms $\left(l-2+\varepsilon_{l}<\mu+\nu+k_{n}-p+t \pi / \alpha^{*}<l-1+\dot{\varepsilon}_{l}, p, c, \kappa\right.$ as in $\left.\left.c\right)\right)$. Furthermore,

$$
P_{l}^{(0)}(f)=\sum_{\mu+\nu=l-1} \frac{1}{\mu ! \nu !}\left(\mathcal{K} f_{\mu \nu}\right) x_{1}^{\mu} x_{2}^{\nu}
$$

is also a term of the form (3.4) where $\kappa=(0), t=p=|\gamma|=0$ and $c=f_{\mu \nu} \epsilon$ $W^{l+k-1-\mu-\nu+e}(M)$. By Lemma 6 there exists a function $w$ which is a finite sum of terms $\widehat{c}(x) \partial_{y}^{\gamma} \partial_{z}^{p}\left\{z^{\mu+t \pi / \alpha} \bar{z}^{\nu} S_{\kappa}(z, \alpha)\right\}$ and conjugate terms with $p \leq \mu+\nu-1, \kappa=$ $\left(k_{0}, \ldots, k_{n}\right) \in \mathcal{K}_{l}\left(\alpha^{*}\right), l+\varepsilon_{l}<\mu+\nu+k_{n}-p+t \pi / \alpha^{*}<l+1+\varepsilon_{l}$,

$$
\widehat{c}=\mathcal{K} c, \quad c \in W^{l+k+1+|\gamma|-\mu-\nu-k_{n}+p+e_{1}-t \pi / \alpha}(M)
$$

such that

$$
\begin{aligned}
w & =0 \quad \text { on } \partial \mathcal{D} \cap \mathcal{V}_{0} \\
L w-P_{l}(f)+L \Sigma_{l-1} & =L w-P_{l}^{(0)}(f)-\left(P_{l-1}(f)-L \Sigma_{l-1}\right)=B_{1}+B_{2}
\end{aligned}
$$

where $B_{2} \in V_{-e^{\prime}}^{l+k}(\mathcal{D})$ and ${ }^{\prime} B_{1}$ is a finite sum of expressions

$$
a(x) \widehat{c} \partial_{y}^{\gamma} \partial_{z}^{p}\left\{z^{\mu+t \pi / \alpha} \bar{z}^{\nu} S_{\kappa}(z, \alpha)\right\}
$$

and conjugate terms $\left(\kappa=\left(k_{0}, \ldots, k_{n}\right) \in \mathcal{K}_{l}\left(\alpha^{*}\right), p \leq \mu+\nu+1, l-1+\varepsilon^{\prime}<\mu+\nu+k_{n}-\right.$ $\left.p+t \pi / \alpha^{*}<l+k-1+\varepsilon^{\prime}, \widehat{c}=\mathcal{K} c, c \in W^{l+k-1+|\gamma|-\mu-\nu+k_{n}+p+e^{\prime}-t \pi / \alpha}\right)$. In particular,

$$
\dot{D}_{y}^{\beta}\left(L w-P_{l}(f)+L \Sigma_{l-1}\right) \in V_{-e_{l}+k-|\beta|}^{l+k-|\beta|}(\mathcal{D}) \quad \text { for }|\beta| \leq k .
$$

Moreover; from the properties of the extension operator $\mathcal{K}$ it follows that

$$
D_{y}^{\beta} w \in V_{-\varepsilon_{1}+k+1-|\beta|}^{1+k+2-|\beta|}(D) \quad \text { for }|\beta| \leq k+1 .
$$

Consequently,

$$
\begin{aligned}
D_{y}^{\beta}\left(u_{l-1}-w\right) & \in V_{-c_{1}+k+1-|\beta|}^{l+k+2-|\beta|}(\mathcal{D}) & & \text { for }|\beta| \leq k+1 \\
u_{l-1}-w & =0 & & \text { on } \partial \mathcal{D} \cap \mathcal{V}_{0}
\end{aligned}
$$


and

$$
\begin{aligned}
D_{y}^{\beta} L\left(u_{l-1}-w\right) & =D_{y}^{\beta}\left(f-P_{l}(f)\right)-D_{y}^{\beta}\left(L w-P_{l}(f)-L \Sigma_{l-1}\right) \\
& \in V_{-e_{l}+k-|\beta|}^{l+k \mid \beta)}(\mathcal{D}) .
\end{aligned}
$$

Let $I_{l}$ denote the set $I_{l}=\left\{t \in \mathbb{N}: l+\varepsilon<t \pi / \alpha^{*}<l+1+\varepsilon\right\}$. Then Lemma 10 yields

$$
u_{l-1}-w=\Sigma^{\prime}+u_{l}
$$

where $\Sigma^{\prime}=\sum_{t \in I_{t}} \widehat{c_{t}}(x) I m z^{t \pi / \alpha(y)}, D_{y}^{\beta} u_{l} \in V_{-e^{\prime}+k+1-|\beta|}^{t+k+3-|\beta|}(\mathcal{D})$ for $|\beta| \leq k, \widehat{c_{t}}=\mathcal{K} c_{t}$, $c_{t} \in W^{l+k+1+e^{\prime}-t \pi / \alpha(\cdot)}(M)$. Furthermore, for $\Sigma_{l}=\Sigma_{l-1}+\Sigma^{\prime}+w$ we get

$$
L \Sigma_{l}-P_{l}(f)=\left(L \Sigma_{l-1}-P_{l}(f)+L w\right)+L \Sigma^{\prime}=C_{1}+C_{2}
$$

where $C_{2} \in V_{-e^{\prime}}^{l+k}(\mathcal{D})$ and $C_{1}$ is a finite sum of expressions of the form (3.5). This proves the proposition

Now we can formulate the main theorem of this paper for $\alpha^{*} \neq \pi, \alpha^{*} \neq 2 \pi$ as a special case of Proposition 4.

Theorem 2. Let $\alpha^{*} \neq \pi, \alpha^{*} \neq 2 \pi$ and let $u \in \dot{W}^{1}(G)$ be a solution of problem (9.1) with $f \in W_{-e}^{l}(G)$. Then $u$ admits the decomposition

$$
u=\Sigma_{l}+u_{l}
$$

in $G \cap \mathcal{V}_{0}$ where $u_{l} \in V_{-e^{\prime}}^{l+2}\left(G \cap \mathcal{V}_{0}\right)$ and $\sum_{l}$ is a finite sum of expressions

$$
\widehat{c}(x) \partial_{y}^{\gamma}\left\{z^{\mu+t \pi / \alpha(y)} \bar{z}^{\nu} S_{\kappa}(z, \alpha(y))\right\}
$$

and conjugate terms. Here $\mu, \nu$ are integers, $\mu \geq 0, \mu+\nu \geq 0, \kappa=\left(k_{0}, \ldots, k_{n}\right) \in \mathcal{K}_{l}\left(\alpha^{*}\right)$, $\mu+\nu+k_{n}+t \pi / \alpha^{*}<l+1+\varepsilon^{\prime}$ and

$$
\widehat{c}=\mathcal{K} c, \quad c \in W^{l+1-\mu-\nu-k_{n}+|\gamma|+\varepsilon^{\prime}-t \pi / \alpha(\cdot)}(M)
$$

If $f \in W_{-e}^{2 l+2}(G)$, then the coefficients $\hat{c}$ in (9.6) may be replaced by their traces which are functions from $W^{2 l+3-\mu-\nu-k_{n}+e^{\prime}-t \pi / \alpha(\cdot)}(M)$.

In the same way one can prove the analogous result for the critical angles $\alpha^{*}=\pi$ and $\alpha^{*}=2 \pi$ by means of Proposition 3.

Theorem 3. Let $\alpha^{*}=\pi$ or $\alpha^{*}=2 \pi$ and let $u \in \dot{W}^{1}(G)$ be a solution of problem (S.1) with $f \in W_{-e}^{l}(G)$. Then $u$ admits the decomposition

$$
u=\Sigma_{l}+u_{l}
$$

in $G \cap \mathcal{V}_{0}$ where $u_{l} \in V_{-e^{\prime}}^{l+2}(G)$ and $\Sigma_{l}$ is a finite sum of expressions of the form

$$
\widehat{c}(x) \partial_{y}^{\gamma}\left\{z^{\mu+t \pi / \alpha} \bar{z}^{\nu} S_{n}(z, \alpha)\right\}
$$


and conjugate terms. Here $n, t, \mu, \nu$ are integer numbers with $n \geq 0, t \geq 0, \nu \geq 0$, $\mu+\nu \geq 0, \mu+\nu+n+t \pi / \alpha^{*}<l+1-\varepsilon^{\prime}$ and $\widehat{c}=\mathcal{K}_{c}$ is the extension of a function

$$
c \in W^{\prime+1+|\gamma|+\varepsilon^{\prime}-n-\mu-\nu-t \pi / a^{\circ}}(M) \text {. }
$$

Remark 2. If $\alpha^{*}=\pi$ or $\alpha^{*}=2 \pi$ and the function $\omega$ in the definition of the dihedron $\mathcal{D}$ does not depend on the variable $y$ (i.e. $\omega(r, y)=\alpha(y)$ ), then the sum $\Sigma_{l}$ in Theorem 3 consists only of terms of the form

$$
\widehat{c}(x) \partial_{y}^{\gamma} z^{\mu+t \pi / \alpha} \bar{z}^{\nu} \quad \text { and } \quad \widehat{C}(x) \partial_{y}^{\gamma} \bar{z}^{\mu+t \pi / \alpha} z^{\nu}
$$

(cf Remark 1), i.e. the asymptotics of $u$ does not differ from that for non-critical angles (see, e.g., $[3,9,10,14])$.

\section{Asymptotics near the vertex of a cube}

In this Section we will investigate the edge asymptotics of a solution $u$ of the Dirichlet problem

$$
L(x, D) u=f \quad \text { in } G, \quad u=0 \text { on } \partial G
$$

if the critical angle $\alpha^{*}=\pi / 2$ occurs in the vertex of a polyhedron. Suppose that the domain $G$ coincides with the infinite cube $K=(0, \infty)^{3}$ in a neighbourhood of the origin and that the principal part $L_{0}(O, D)$ of $L$ with coefficients frozen in the origin is equal to the Laplacian. We introduce the following weighted Sobolev spaces in $K$. For given integer $l \geq 0$ and real $\beta, \gamma$ we define $V_{\beta, \gamma}^{l}(K)$ as the closure of $C_{0}^{\infty}(\bar{K} \backslash \mathcal{S})$ with respect to the norm

$$
\|u\|=\left(\sum_{|\alpha| \leq l} \int_{K} \rho^{2 \beta}\left(\prod_{j=1}^{3} \frac{r_{j}}{\rho}\right)^{2 \gamma} r^{2(|\alpha|-l)}\left|D^{\alpha} u\right|^{2} d x\right)^{1 / 2} .
$$

Here $\rho=|x|, r_{j}(x)$ denotes the distance of $x$ to the $x_{j}$-axis $(j=1,2,3)$ and $r(x)=$ $\min _{j} r_{j}(x)$ denotes the distance of $x$ to the set $S$ of the edge points of $K$. Furthermore, $W_{\beta, \gamma}^{l}(K)^{\prime}(\beta>-3 / 2, \gamma>-1)$ will be defined as the closure of $C^{\infty}(\bar{K})$ with respect to the norm

$$
\|u\|=\left(\sum_{|\alpha| \leq l} \int_{K} \rho^{2 \beta}\left(\prod_{j=1}^{3} \frac{r_{j}}{\rho}\right)^{2 \gamma}\left|D^{\alpha} u\right|^{2} d x\right)^{1 / 2}
$$

Let $\chi_{1} \in C^{\infty}([0, \infty)) ; \chi \in C^{\infty}((0, \pi / 2))$ be smooth cut-off functions with support in $[0,1]$ which are equal to one in $[0,1 / 2]$. Furthermore, let $\chi$ be the cut-off function in $K$ which is defined by the equation

$$
\chi(x)=\chi_{1}(\rho) \chi_{2}(\vartheta)
$$

where $\rho, \phi, \vartheta$ denote the spherical coordinates in $\mathbb{R}^{3}\left(\rho=|x|, \phi=\arg \left(x_{1}+i x_{2}\right)\right.$, $\left.\cos \vartheta=x_{3} /|x|\right)$. 
We assume that the solution $u$ of problem (4.1) belongs to the Sobolev space $W^{1}(G)$ and that the right-hand side $f$ of (4.1) satisfies the condition $r^{-\varepsilon} f \in L_{2}(G)$, $r^{-e} \partial f / \partial x_{j} \in L_{2}(G)(j=1,2,3 ; \varepsilon>1 / 2)$. Then $\chi u \in V_{0,0}^{1}(K)$ and $\chi f \in W_{-e,-e}^{1}(K)$. Since $u(x)=x_{1} x_{2} x_{3}=\rho^{2} \sin \phi \cos \phi \sin ^{2} \vartheta \cos \vartheta$ is a solution of the problem $\Delta u=0$ in $K, u=0$ on $\partial K$ the function $g(\phi, \vartheta)=\sin \phi \cos \phi \sin ^{2} \vartheta \cos \vartheta$ is a positive eigenfunction to the eigenvalue $\lambda=3$ of the operator $\delta+\lambda^{2}+\lambda$ ( $\delta$ denotes the Laplace-Beltrami operator on the unit sphere in $\mathbb{I R}^{3}$ ) with the boundary conditions $\left.g\right|_{\phi=0}=\left.g\right|_{\phi=\pi / 2}=$ $\left.g\right|_{\delta=\pi / 2}=0$. Using the fact that the only positive eigenfunction of $-\delta$ belongs to the first eigenvalue one gets

$$
v=\chi^{u} \in V_{-e, e^{\prime}}^{3}(K) \quad \text { and } \quad f_{1}=L(\chi u) \in W_{-\varepsilon,-\varepsilon}^{1}(K)
$$

(see [12: Theorem A2]) where $\varepsilon^{\prime}$ is an arbitrary positive real number larger than $2-\pi / \alpha$. Here $\alpha$ is a function of the variable $x_{3}$ which arises from the transformation

$$
\begin{aligned}
& x_{1}^{\prime}=\left(a_{0,2,0}\left(0,0, x_{3}\right) D\right)^{1 / 2} x_{1}-\frac{1}{2} a_{1,1,0}\left(0,0, x_{3}\right)\left(a_{0,2,0}\left(0,0, x_{3}\right) D\right)^{-1 / 2} x_{2} \\
& x_{2}^{\prime}=a_{0,2,0}\left(0,0, x_{3}\right)^{-1 / 2} x_{2} \\
& x_{3}^{\prime}=x_{3}
\end{aligned}
$$

$\left(D=a_{2,0,0}\left(0,0, x_{3}\right) a_{0,2,0}\left(0,0, x_{3}\right)-\frac{1}{4} a_{1,1,0}\left(0,0, x_{3}\right)\right)$. Applying this transformation to the equations

$$
L v=f_{1} \quad \text { in } K, \quad v=0 \text { on } \partial K
$$

one gets the Dirichlet problem

$$
L^{\prime} v=f_{1} \quad \text { in } K^{\prime}, \quad v=0 \text { on } \partial K^{\prime}
$$

where

$$
K^{\prime}=\left\{x^{\prime}=\left(x_{1}^{\prime}, x_{2}^{\prime}, x_{3}^{\prime}\right): 0<x_{3}^{\prime}<\infty, 0<\phi^{\prime}=\arg \left(x_{1}^{\prime}+i x_{2}^{\prime}\right)<\alpha\left(x^{\prime}{ }_{3}\right)\right\}
$$

and the coefficients of $L^{\prime}=\sum_{i+j+k \leq 2} b_{i j k}\left(x^{\prime}\right) \partial_{x_{1}^{\prime}}^{i} \partial_{x_{2}}^{j} \partial_{x^{\prime}}^{i}$ are smooth and satisfy the condition

$$
b_{2,0,0}\left(0,0, x^{\prime}{ }_{3}\right) \equiv b_{0,2,0}\left(0,0, x^{\prime}{ }_{3}\right) \equiv 1, \quad b_{1,1,0}\left(0,0, x^{\prime}\right) \equiv 0
$$

Obviously, $\alpha(0)=\pi / 2$. Suppose that the function $\alpha$ satisfies the inequality $\sup (2-$ $\pi / \alpha)<\inf (2-\pi / \alpha)+\varepsilon$. We want to use the results of Section 3 . Therefore, we introduce the coordinates

$$
\xi_{1}=x^{\prime}{ }_{1} / x^{\prime}{ }_{3}, \quad \xi_{2}=x^{\prime}{ }_{2} / x^{\prime}{ }_{3}, \quad t=\log x_{3}^{\prime} .
$$

Then the domain $K^{\prime}$ corresponds to the dihedron

$$
\mathcal{D}=\left\{\left(\xi_{1}, \xi_{2}, t\right) \in \mathbb{R}^{3}: t \in \mathbb{R}, 0<\phi^{\prime}=\arg \left(\xi_{1}+i \xi_{2}\right)<\alpha\left(e^{i}\right)\right\}
$$


in these new coordinates. If we set $w=e^{-(e+3 / 2) t} v$, then the function $w$ is a solution of the Dirichlet problem

$$
L^{\prime \prime} w=e^{-(e-1 / 2) \mathfrak{t}} f_{1}=F \quad \text { in } \mathcal{D}, \quad w=0 \text { on } \partial \mathcal{D}
$$

where $L^{\prime \prime}=\sum_{i+j+k \leq 2} c_{i j k}\left(\xi_{1}, \xi_{2}, t\right) \partial_{\xi_{1}}^{i} \partial_{\xi_{2}}^{j} \partial_{i}^{k}$ is a second order differential operator with smooth coefficients satisfying the condition (4.3), i.e.

$$
c_{2,0,0}(0,0, t) \equiv c_{0,2,0}(0,0, t) \equiv 1, \quad c_{1,1,0}(0,0, t) \equiv 0 .
$$

Lemma 11. Let $\psi$ be a function in $K^{\prime}$ satisfying the condition

$$
\psi\left(x^{\prime}\right)=0 \quad \text { for } \frac{r^{\prime}}{\rho^{\prime}}=\left[\frac{x_{1}^{\prime 2}+x_{2}^{\prime 2}}{x_{1}^{\prime 2}+x_{2}^{\prime 2}+x_{3}^{\prime 2}}\right]^{1 / 2}>1 / 2
$$

Then

$$
\psi \in V_{\beta, \gamma}^{l}\left(K^{\prime}\right) \quad \text { if and only if } \quad e^{(\beta-1+3 / 2) t} \psi \in V_{\gamma}^{l}(\mathcal{D}) .
$$

Lemma 11 implies that the solution $w$ of problem (4.4) belongs to $V_{-e^{\prime}}(\mathcal{D})$. Furthermore, one can show that $F=e^{-(\varepsilon-1 / 2) t} f_{1} \in W_{-e}^{1}(\mathcal{D})$ if $f_{1} \in W_{-\varepsilon,-e}^{1}\left(K^{\prime}\right)$. The connection between the spaces $V_{\beta, \gamma}^{\prime}\left(K^{\prime}\right)$ and $V_{\gamma}^{\prime}(\mathcal{D})$ in Lemma 11 can be used to define weighted Sobolev spaces on the $x_{3}$-axis. We define $V_{\beta}^{\prime}\left(\mathbb{R}_{+}\right)(\beta, s \in \mathbb{R}, s>0)$ as the set of all functions $g$ on $\mathbb{R}_{+}$for which the function

$$
g_{1}(t)=e^{(\beta-s+1 / 2) t} g\left(e^{t}\right)
$$

belongs to the Sobolev space $W^{s}(\mathbb{R})$. For integer $s$ the norm in $V_{\beta}^{s}\left(\mathbb{R}_{+}\right)$is equivalent to the norm

$$
\|g\|=\left(\sum_{j=0}^{s} \int_{0}^{\infty} x_{3}^{2(\beta-s+j)}\left|D_{x_{3}}^{j} g\left(x_{3}\right)\right|^{2} d x_{3}\right)^{1 / 2}
$$

Proposition 5. Let $u \in \dot{W}^{1}(G)$ be a solution of problem (4.1) where $r^{- \text {e }} f \in L_{2}(G)$ and $r^{-e} \partial f / \partial x_{j} \in L_{2}(G)(j=1,2,3)$ and $\varepsilon$ is a real number less than $\frac{1}{2}$ satisfying the inequality $\sup \left(2-\pi / \alpha\left(x_{3}\right)\right)<\inf \left(2-\pi / \alpha\left(x_{3}\right)\right)+\varepsilon$. Then $\chi u$ admits the decomposition

$$
\begin{aligned}
\chi u= & \hat{d}_{1}\left(x^{\prime}\right) r^{\prime \pi / \alpha\left(x_{3}\right)} \sin \left(\frac{\pi}{\alpha\left(x_{3}\right)} \dot{\phi}^{\prime}\right) \\
& +\hat{d}_{2}\left(x^{\prime}\right)\left(r^{\prime 2}\left(1-\cos 2 \phi^{\prime}\right)+\frac{\left(\alpha\left(x_{3}\right)-\pi / 2\right)\left(\cos \left(2 \alpha\left(x_{3}\right)\right)-1\right)}{\sin \left(2 \alpha\left(x_{3}\right)\right)}\right. \\
& \left.\times \frac{r^{\prime} \sin 2 \phi^{\prime}-x_{3}^{2-\pi / \alpha\left(x_{3}\right)} r^{\prime \pi / \alpha\left(x_{3}\right)} \sin \left(\pi \phi^{\prime} / \alpha\left(x_{3}\right)\right)}{\alpha\left(x_{3}\right)-\pi / 2}\right)+u^{\prime}
\end{aligned}
$$

where $u^{\prime} \in V_{-\varepsilon,-e+\delta}^{3}(K), \hat{d}_{1}$ is an extension of a function $d_{1} \in V_{-\delta}^{2+e-\delta-\pi / a}\left(\mathbb{R}_{+}\right)$, $d=d\left(x_{3}\right)$, and $\hat{d}_{2}$ is an extension of the trace $\left.f_{1}\right|_{x_{1}=x_{2}=0} \in V_{0}^{\epsilon}\left(\mathbb{R}_{+}\right)$into the domain 
$K^{\prime}$. Here $r^{\prime}=\left({x^{\prime}}_{1}^{2}+{x^{\prime}}_{2}^{2}\right)^{1 / 2}$ and $\phi^{\prime}=\arg \left(x_{1}^{\prime}+i x_{2}^{\prime}\right)$ are the polar coordinates in the $\left(x_{1}^{\prime}, x^{\prime}{ }_{2}\right)$-plane and $\delta$ is an arbitrary number between $\sup (2-\pi / \alpha)$ and $\inf (2-\pi / \alpha)+\varepsilon$.

Proof. Applying Theorem 2 to problem (4.4) one gets

$$
\begin{aligned}
w= & \hat{c_{1}}(\xi, t) r_{\xi}^{\pi / \alpha\left(e^{t}\right)} \sin \left(\pi \phi^{\prime} / \alpha\left(e^{t}\right)\right) \\
& +\frac{1}{4}\left(\mathcal{K} F_{0}\right)(\xi, t)\left(r_{\xi}^{2}\left(1-\cos 2 \phi^{\prime}\right)+\frac{(\alpha-\pi / 2)\left(\cos \left(2 \alpha\left(e^{t}\right)\right)-1\right)}{\sin \left(2 \alpha\left(e^{t}\right)\right)}\right. \\
& \left.\times \frac{r_{\xi}^{2} \sin 2 \phi^{\prime}-r_{\xi}^{\pi / \alpha\left(e^{t}\right)} \sin \left(\pi \phi^{\prime} / \alpha\left(e^{t}\right)\right)}{\alpha\left(e^{t}\right)-\pi / 2}\right)+w^{\prime} .
\end{aligned}
$$

Here $w^{\prime} \in V_{-\epsilon+\delta}^{3}(\mathcal{D}), r_{\xi}=\left(\xi_{1}^{2}+\xi_{2}^{2}\right)=r^{\prime} / x_{3}, \phi^{\prime}=\arg \left(\xi_{1}+i \xi_{2}\right)=\arg \left(x_{1}^{\prime}+i x_{2}^{\prime}\right)$, $\widehat{c_{1}}(\xi, t)=\mathcal{K} c_{1}$ is an extension of a function $c_{1} \in W^{2+\varepsilon-\delta-\pi / \alpha}(\mathbb{R})$ and $F_{0}$ is the trace of $F$ on the edge of $\mathcal{D}$. Multiplying (4.6) by $e^{(e+3 / 2) t}=x_{3}^{e+3 / 2}$ one gets (4.5) where

$$
\begin{aligned}
\hat{d_{1}}\left(x^{\prime}\right) & =x_{3}^{e+3 / 2-\pi / \alpha\left(x_{3}\right)} \hat{c_{1}}(\xi, t)=x_{3}^{e+3 / 2-\pi / \alpha} \widehat{c_{1}}\left(\frac{x_{1}^{\prime}}{x_{3}}, \frac{x_{2}^{\prime}}{x_{3}}, \log x_{3}\right) \\
\hat{d_{2}} & =\frac{1}{4} x_{3}^{e-1 / 2}\left(\mathcal{K} F_{0}\right)(\xi, t) \ldots
\end{aligned}
$$

This proves the proposition

\section{References}

[1] Costabel, M. and M. Dauge: Edge asymptotics on a skew cylinder. Lecture given at the International Workshop "Analysis in domains and on manifolds with singularities", Breitenbrunn (Germany) 1990.

[2] Costabel, M. and M. Dauge: General edge asymptotics of solutions of second order elliptic boundary value problems, I and II. Preprint. Paris: Université Pierre et Marie Curie 1991, Preprints R91016 and R91017.

[3] Dauge, M.: Elliptic boundary value problems in corner domains. Lect. Notes Math. 1341 (1988) 1 - 257.

[4] Kondratjev, V.A.: Boundary value problems for elliptic equations in domains with conical or angular points. Trudy Moskov. Mat. Obšč. 16 (1967), 209 - 292.

[5] Kondratjev, V.A.: Singularities of solutions of the Dirichlet problem for elliptic equations of second order in a neighbourhood of an edge. Diff. Uravn. 13 (1977), 2026 - 2032.

[6] Maz'ya, V.G. and B.A. Plamenevskij: On elliptic boundary value problems in domains with piece-wise smooth boundaries. In: Lectures at the symposium on fluid mechanics and related problems of analysis 1971. Tbilissi: Mezniereba 1973, pp. 171 - 182.

[7] Maz'ya, V.G. and B.A. Plamenevskij: Estimates in $L_{p}$ and Hölder classes and the Miranda -Agmon maximum principle for solutions of elliptic boundary value problems in domains with singular points on the boundary. Math. Nachr. 81 (1978), 25 - 82.

[8] Maz'ya, V.G: and B.A. Plamenevskij: $L_{p}$-estimates for solutions of elliptic boundary value problems in domains with edges. Trudy Moskov. Mat. Obšć. 37 (1978), 49- 93. 
[9] Maz'ya, V.G. and J. Rossmann: Über die Lösbarkeit und die Asymptotik der Lösungen elliptischer Randwertaufgaben in Gebieten mit Kanten I-III. Preprints 07/84, 30/84 and 31/84. Berlin: Inst. für Math. I: pp. 1 - 50, II: pp. 1 - 50, III: pp. 1 - 44.

[10] Maz'ya, V.G. and J. Rossmann: Über die Asymptotik der Lösungen elliptischer Randuertaufgaben in der Umgebung von Kanten. Math. Nachr. 138 (1988), 27 - 53.

[11] Maz'ya, V.G. and J. Rossmann: On the arymptotics of solutions to the Dirichlet problem for second order elliptic equations in domains with critical angles on the edges. Preprint Lith-Math-R-91-37. Linköping: University, Dep. of Math. 1991, pp 1 - 38.

[12] Maz'ya, V.G. and J. Rossmann: On the Agmon-Miranda maximum principle for solutions of elliptic equations in polyhedral and polygonal domains. Ann. Global Anal. Geom. 9 (1991), 253 - 303.

[13] Maz'ya, V.G. and J. Rossmann: On a problem of Babuska (Stable asymptotics of the solution to the Dirichlet problem for elliptic equations of second order in domains with angular points) Math. Nachr. 155 (1992), 199 - 220.

[14] Nikiškin, V.A.: Singularities of the solution of the Dirichlet problem for elliptic differential equations of second order in a neighbourhood of edges. Vestnik Moskov. Univ., Matem. 34 (1979), 51 - 62.

[15] v. Petersdorff, T. and E.P. Stephan: Decomposition in edge and comer singularities for the solution of the Dirichlet problem of the Laplacian in a polyhedron. Math. Nachr. 149(1990), 71 - 104.

[16] Rempel, S. and B.-W. Schulze: Asymptotics for mixed boundary value problems. Berlin: Akademie-Verlag 1989.

[17] Rossmann, J.: The asymptotics of solutions of linear elliptic variational problems in domains with edges. Z. Anal. Anw. 9 (1990), 565 - 578.

[18] Schulze, B.-W.: Regularity with continuous and branching arymptotics for elliptic operators on manifolds with edges. Int. Equ. Oper. Theory 11 (1988), 557 - 602.

[19] Schulze, B.-W.: Pseudo-Differential Operators on Manifolds with Singularities. New York and North-Holland: Elsevier 1991. 